\title{
Energy-efficient design of two-tier femtocell networks
}

\author{
Ying Wang ${ }^{*}$, Yuan Zhang ${ }^{\dagger}$, Yongce Chen ${ }^{\dagger}$ and Rong Wei ${ }^{\dagger}$
}

\begin{abstract}
With the exponential increase in mobile internet traffic, future cellular networks face great challenges to satisfy the demand of network capacity. At the same time, high data rate transmission and rapid increasing number of users also seriously burden the power consumption and the cost of cellular networks. Base station (BS) is the main part of power consumption, so reducing energy consumption of the BS can obviously reduce the total energy consumption. This paper investigates the effect on energy efficiency by adopting activity-aware sleeping strategies both in macrocell base station (MBS) and femtocell access point (FAP) in a two-tier femtocell network. By using stochastic geometry, we develop a trade-off between energy saving and coverage extension, regarded as the FAP additional connections by neighboring femtocell user equipment (FUE) or macrocell user equipment (MUE). Specifically, we derive users' coverage probabilities, which is defined as the probability of user connecting to BS, in closed forms with different sleeping strategies and access policies. Moreover, we formulate power consumption minimization and energy efficiency problem and determine the optimal joint MBS-FAP operating regimes. Numerical results show that sleeping scheme and femtocell access mode both have effects on coverage probability and energy efficiency, and the effect of femtocell access mode on coverage probability is greater than sleeping scheme.
\end{abstract}

Keywords: Two-tier femtocell network; Sleeping strategy; Stochastic geometry; Coverage probability; Energy efficiency

\section{Introduction}

Looking ahead to the year 2020 and beyond, there will be explosive growth in mobile data traffic. It is estimated that hotspot traffic may grow up to 1,000 times [1]. Small cells seem to be a reasonable way to cater to the rapid increasing data traffic and provide higher spectral efficiency and energy efficiency.

Generally speaking, small cells such as femtocells, which provide improved radio coverage and throughput by wireless access, operate in three access modes: open access, closed access, and hybrid access. When femtocells work in closed access mode, only registered femtocell user equipments (FUEs) can connect to the femtocell access point (FAP). In open access mode, all kinds of users will be allowed to connect to the FAP. In hybrid access mode, registered users and non-registered macro users that satisfy certain constraints can connect to the FAP.

${ }^{*}$ Correspondence: wangying@bupt.edu.cn

†Equal contributors

State Key Laboratory of Networking and Switching Technology, Beijing

University of Posts and Telecommunications, 100876 Beijing, China
Although there already have some researches on femtocells with partially open channels [2], for simplicity, we consider completely closed access mode and completely open access mode in this paper.

Besides the deployment of small cells, there are several aspects for energy conservation: base stations (BSs), user terminals, and the exploitation of renewable energy. BSs consume nearly $60 \%$ to $80 \%$ of the total energy in cellular networks, thus saving the energy consumption of BSs can implement green network effectively [3]. Macrocell base stations (MBSs) are usually powered on, while traffic demands are different in various time and areas [4], so these unnecessary MBSs waste energy seriously. In addition, in a two-tier femtocell network, to cope with the fierce increase of mobile traffic demand, network operators deploy massive and uncoordinated FAPs, which leads to a considerable increase in energy consumption.

Adopting sleeping mode in these unnecessary BSs is the most direct and effective way for energy conservation, so

\section{Springer}


we employ the method in heterogeneous networks, while the coverage problem caused by such operation cannot be ignored.

In this paper, we use stochastic geometry [5,6] to study the trade-off between coverage extension (i.e., FAP additional connections by neighboring FUE or MUE) and energy saving in two-tier femtocell networks. MBSs and FAPs are set to work in a coordinated sleeping mode, where certain MBSs will be shut off while other active MBSs and FAPs would extend their coverage to avoid coverage hole. Specifically, an activity-aware sleeping strategy for the two-tier femtocell network is proposed. Then, the probabilities of users successfully communicate with BSs, termed users' coverage probabilities, are derived in closed forms with different sleeping strategies and access modes. In addition, energy consumption optimization and energy efficiency problems are proposed. Numerical results prove that adopting sleeping strategy in both MBSs and FAPs can effectively improve energy efficiency, and the gain depends on the strategy and femtocell access mode.

The main contributions of this paper are listed as follows:

- Using stochastic geometry, we analyze the problems of total power consumption and energy efficiency through deploying the activity-aware sleeping strategy in cognitive MBSs and FAPs.

- A concrete analysis on the coverage extension problem is proposed. In particular, expressions for the coverage probabilities with different sleeping policies and access modes are derived in closed forms.

- On the basis of uncertainties such as the activity probabilities of MBS and FAP, the probabilities of $\mathrm{MBS}$ and FAP remain in operation and the minimum total power consumption are derived. Finally, we explore how the activity probabilities of MBSs and FAPs affect energy efficiency.

- The effects of noise and power control used to maintain network coverage are also investigated.

The remainder of this paper is organized as follows: Section 2 discusses related works and Section 3 presents system model. In Section 4, activity-aware sleeping strategy is proposed and the other sleeping strategies are introduced for comparison. In Section 5, coverage probability is derived firstly with different sleeping strategies and femtocell access policies, then the total power consumption is optimized, and energy efficiency is analyzed lastly. Section 6 validates our analysis through simulation results and numerical results, and concluding remarks are given in Section 7.

\section{Related work}

There are mainly two types of methods to decrease the total energy consumption of BSs in heterogeneous networks:

- Turning small cell access points (SAPs) into sleeping mode when they are not serving any users.

- Adopting sleeping mode in MBSs.

For turning SAPs into sleeping mode, [7] introduces energy-efficient sleeping mode algorithms for small cell BSs and discusses three different strategies for algorithm control, e.g., small cell controlled strategy, core network controlled strategy, and user equipment controlled strategy. A trade-off strategy between traffic offloading from the macrocell and the energy consumption of the small cell is proposed in [8], who provides a sleeping strategy for the cognitive SAPs. In [9], authors take SAPs to have cognitive capabilities and define a fundamental limit which allows robust user detection on the interference density. So an energy efficient SAP design is impossible when beyond that limit.

For adopting sleeping mode in MBSs, some researches verify that energy consumption of MBSs can be significantly reduced while maintaining the downlink coverage and user power consumption performance [10]. Soh et al. study the overage extension and energy saving problems in [11], where closed-form expression for macrocell user equipment (MUE) coverage probability is derived. The purpose of MBSs is to maintain network coverage, meanwhile MBSs increase energy consumption tremendously. Only adopting sleeping mode in FAPs can keep coverage while can not effectively reduce system energy consumption. On the other hand, only adopting sleeping mode in MBSs effectively reduces system energy consumption while reduces the network coverage. So adopting sleeping mode in both MBSs and FAPs can reach a tradeoff between coverage and energy consumption.

Recently, some researches focus on this field. Pan et al. investigate an optimal solution of power saving for femtocell cluster deployments in the sleeping mode involved macro-femto two-tier scenarios in [12]. They propose a coverage extension-based energy optimization scheme for femtocell cluster deployments, which enables FAPs to adapt different sleeping patterns when macrocells are involved in sleep activation. Cao et al. [13] control the numbers of $\mathrm{MBS} / \mathrm{micro} \mathrm{BS}$ to solve energy efficiency maximization problem for two-tier heterogeneous cellular networks and propose explicit analytical expressions of cell size distributions. These works study sleeping patterns and coverage extension, while research on the effects of BS sleeping patterns and femtocell access modes on coverage extension and energy consumption remains an open issue. 


\section{System model}

This section introduces the model of a two-tier femtocell network, user access mechanism, and signal to interference plus noise ratio (SINR). Then, power consumption and energy efficiency problems are described.

\subsection{Network model}

A two-tier femtocell network, sharing the same bandwidth consisting of macrocells networks overlaid with femtocells, is shown in Figure 1. The macrocell tier is comprised of MBSs and MUEs. MBSs are modeled as independent homogeneous poisson point process (PPP) of intensity $\lambda_{m}$ and each MBS transmits with a constant power $P$. The probability density function (pdf) of the distance $R_{m}$ between each MBS and its designated MUE pair is given by $f_{R_{m}}(r)=2 \pi r \lambda_{m} \exp \left(-\pi r^{2} \lambda_{m}\right)$ [14]. The femtocell tier consists of FAPs and FUEs. The same model as MBSs with intensity $\lambda_{f}$ and each FAP transmits with a constant power $Q$. The pdf of the distance $R_{f}$ between each FAP and its designated FUE pair is given by $f_{R_{f}}(r)=$ $2 \pi r \lambda_{f} \exp \left(-\pi r^{2} \lambda_{f}\right)$.

Assuming universal frequency reuse among BSs, users are associated with their nearest BS which serves only one user.

\subsection{User access mechanism}

When femtocells operate in closed access mode, only registered FUEs can be allowed to contact to FAPs. Specific analysis of performance evaluation refers to Section 5 . While in open access mode, both MUEs and unregistered FUEs can be allowed to contact to FAPs, then the coverage region of FAP includes FUE and MUE connecting to femtocell. Details can be found from the diagrams in Figure 2.

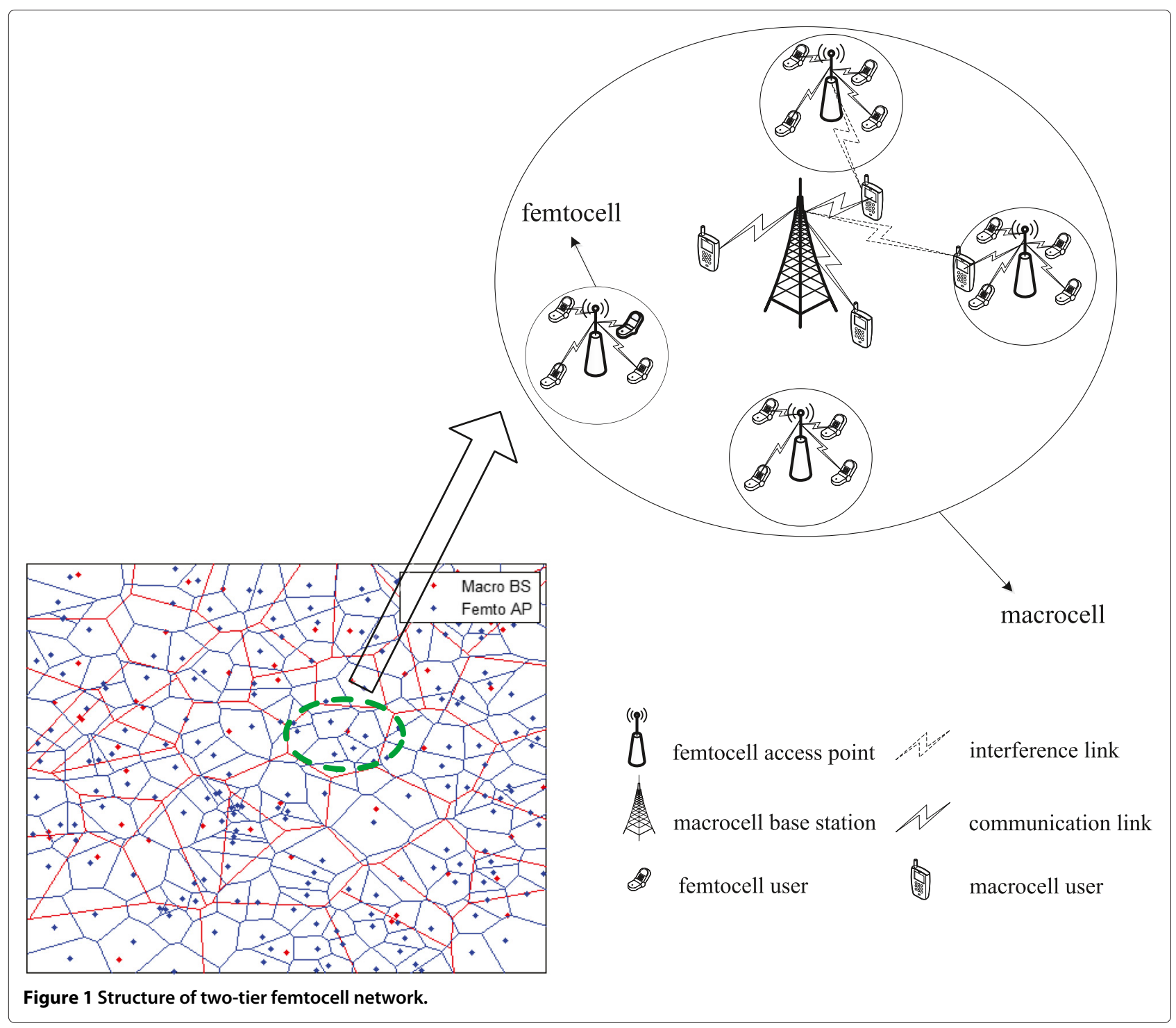


From Figure 2, we can see that when the distance $\kappa r_{m}{ }^{\mathrm{a}}$ between user and the active MBS is shorter than the distance $r_{f}$ between user and the active FAP, then the user communicates with the active MBS. On the contrary, the user communicates with the active FAP.

\subsection{Signal to interference plus noise ratio}

Here, the effect of noise is taken into account. When a user is at the origin 0 , the BS communicating with this user is denoted by its location. For downlink transmission of a BS $\mathbf{x}$ to a user $\mathbf{u}$ at origin 0 , the SINR at a user can be defined as:

$$
\operatorname{SINR}(\mathbf{x} \rightarrow u)=\frac{P_{t, \mathbf{x}} h_{\mathbf{x}} g(\mathbf{x})}{\sum_{\mathbf{y} \in \Theta(\mathbf{x})} P_{t, \mathbf{y}} h_{\mathbf{y}} g(\mathbf{y})+\sigma^{2}},
$$

where $\Theta(\mathbf{x})$ denotes the set of interferers with BS $\mathbf{x}, P_{t, \mathbf{x}}$ is the transmit power of BS, and $h_{\mathbf{x}}, h_{\mathbf{y}}$ are the channel power gain between BS $(\mathbf{x}, \mathbf{y})$ and its users, respectively.

We assume that $h_{\mathbf{x}} \sim \exp (1)$ and $h_{\mathbf{y}} \sim \exp (1)$, the background noise is additive white Gaussian with variance $\sigma^{2}$. The path loss function is denoted by $g(\mathbf{x})=\|\mathbf{x}\|^{-\alpha}$, and $\alpha$ is the path loss exponent.

\subsection{Power consumption model}

In a two-tier femtocell network, the total power consumption comes from macrocell tier and femtocell tier, which is expressed as:

$$
P_{\text {tot }}=\lambda_{m}\left(P_{m 0}+\Delta M \beta_{m} P\right)+\lambda_{f}\left(P_{f 0}+\Delta f Q\right),
$$

where $P_{m 0}$ and $P_{f 0}$ are the static power expenditure of the MBS and FAP, and $\Delta M$ and $\Delta f$ are the slope of the load-dependent power consumption in MBS and FAP, respectively. For the case of macrocell tier, to maintain the total coverage area after switching off some BSs, we adopt the fixed power control policy [11] and assume that all active MBSs transmit with power $\beta_{m} P$, where $\beta_{m}$ is the power control coefficient of MBS. While for the case of dense deployment of FAPs, certain users need to connect to FAP located further away and experience lower amount of inter-cell interference, so it may be believed that the coverage probability is independent of the sleeping mode [15]. So active FAP also transmits with power $Q$.

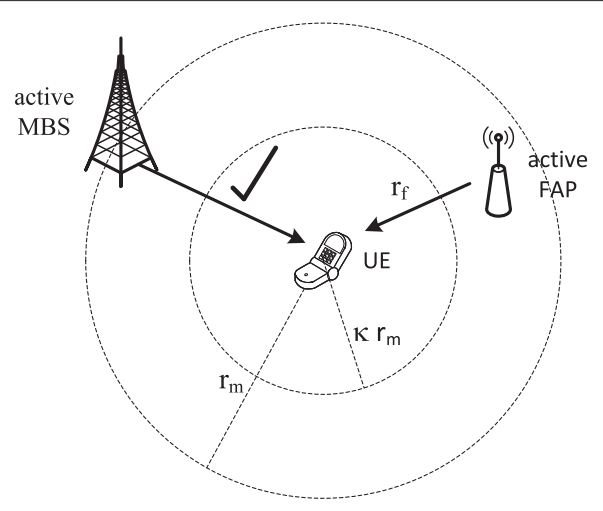

UE communicates with MBS

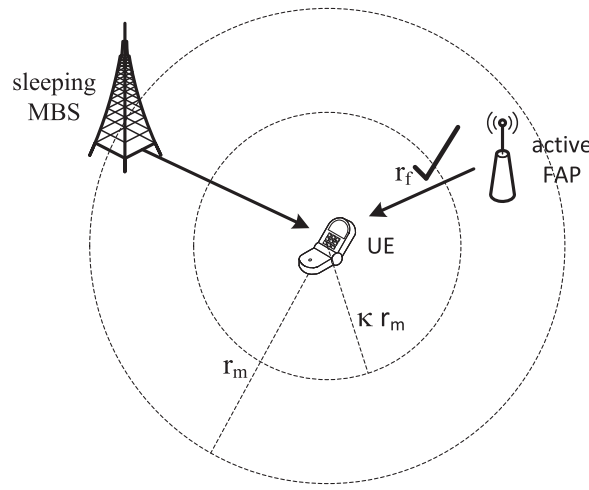

UE communicates with FAP

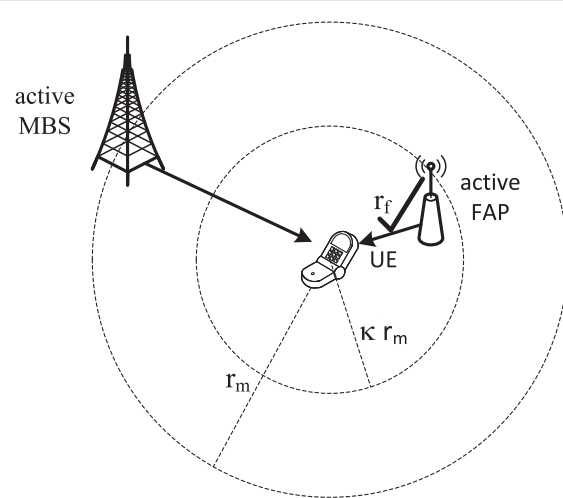

UE communicates with FAP

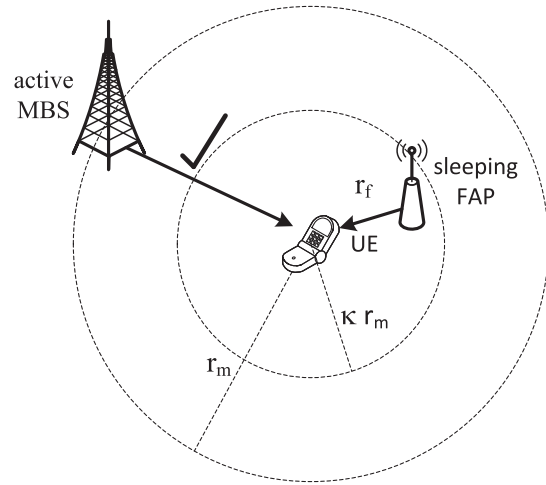

UE communicates with MBS

Figure $\mathbf{2}$ User selection strategy with the activity level of BSs and location of users. 


\subsection{Energy efficiency}

Here, energy efficiency can be expressed in terms of the radio of area spectral efficiency (network throughput, $\left.T_{\text {tot }}\right)$ and network power consumption $\left(P_{\text {tot }}\right)$ as:

$$
\eta=\frac{T_{\text {tot }}}{P_{\text {tot }}}
$$

The coverage probability of MUE and FUE are given by $P_{m}$ and $P_{f}$, respectively. $T_{\text {tot }}$ is taken as $T_{\text {tot }}=T_{m}+T_{f}=$ $\lambda_{m} P_{m} \log _{2}\left(1+\gamma_{m}\right)+\lambda_{f} P_{f} \log _{2}\left(1+\gamma_{f}\right)$, where $\gamma_{m}$ and $\gamma_{f}$ denote SINR thresholds of macro tier and femto tier, respectively.

\section{Base station sleeping strategies}

In this section, we consider two different sleeping policies, namely, random sleeping strategy and activity-aware sleeping strategy, which is based on the network activity level (can be regarded as load or traffic demands). Specifically, the analysis on policies with different femtocell access modes are different. Details refer to the following.

\subsection{Random sleeping strategy}

Random sleeping strategy, we take it as a Bernoulli trial, that is each BS actives with probability $q$ and sleeps with probability $1-q$ independently. In detail, every MBS (or FAP) actives with probability $q_{m}$ (or $q_{f}$ ) and sleeps with probability $1-q_{m}$ (or $1-q_{f}$ ) independently, and the MBSs (or FAPs) intensity is $\lambda_{m} q_{m}$ (or $\lambda_{f} q_{f}$ ) [11]. When femtocell operates in closed access mode, the pdf of the distance $R_{m}$ (or $R_{f}$ ) between each MBS (or FAP) and its designated MUE (or FUE) pair is given by $2 \pi r \lambda_{m} q_{m} \exp \left(-\pi r^{2} \lambda_{m} q_{m}\right)$ (or $2 \pi r \lambda_{f} q_{f} \exp \left(-\pi r^{2} \lambda_{f} q_{f}\right)$ ). When femtocell operates in open access mode, the pdf of the distance $R_{m}$ (or $R_{f}$ ) between each MBS (or FAP) and its designated MUE (or FUE) pair is given by $2 \pi r\left(\lambda_{m} q_{m}+k^{2} \lambda_{f} q_{f}\right) \exp \left(-\pi r^{2}\left(\lambda_{m} q_{m}+k^{2} \lambda_{f} q_{f}\right)\right)$ (or $\left.2 \pi r\left(\lambda_{m} / k^{2} q_{m}+\lambda_{f} q_{f}\right) \exp \left(-\pi r^{2}\left(\lambda_{m} / k^{2} q_{m}+\lambda_{f} q_{f}\right)\right)\right)$ [14].

\subsection{Activity-aware sleeping strategy}

Unlike random sleeping strategy described above, the strategy introduced in this subsection is mainly based on the activity level of networks. Being different from [11], the proposed strategy, activity-aware sleeping strategy, is suitable for the two-tier femtocell network, also taking consideration into the access mode of femtocells and the location of users. That is to say, when femtocells operate in closed access mode, sleeping control and user access only depend on the activity level of networks; while for open access mode, besides the activity level of networks, user access still depends on the location of users.

Relevant parameters are set as follows: $A$ denotes the random activity of BS within a Poisson-Voronoi cell and takes values in [0,1], $A_{i}$ represents user activity within the cell that a BS covers, and $A_{i}$ and $A$ satisfy $A_{i} \sim A$. That is to say, any user located in the Poisson-Voronoi cell with activity level $a$ would connect to the BS with probability a. Then, we model this strategic sleeping as a function s: $[0,1] \rightarrow[0,1]$, in other words, when BS has activity level $x$, then the BS operates with probability $s(x)$ and sleep with probability $1-s(x)$, respectively. The representations of different layers distinguish with each other with the different subscripts.

Specifically, for macrocell tier (or femtocell tier), every MBS (or FAP) actives with probability $E\left\{s_{m}\right\}$ (or $E\left\{s_{f}\right\}$ ) and sleeps with probability $1-E\left\{s_{m}\right\}$ (or $1-E\left\{s_{f}\right\}$ ) independently, and the MBSs (or FAP) intensity is $\lambda_{m} E\left\{s_{m}\right\}$ (or $\lambda_{f} E\left\{s_{f}\right\}$ ). When femtocell operates in closed access mode, the pdf of the distance $R_{m}^{\prime}$ (or $R_{f}^{\prime}$ ) between each MBS (or FAP) and its designated MUE (or FUE) pair is given by $2 \pi r \lambda_{m} E\left\{s_{m}\right\} \exp \left(-\pi r^{2} \lambda_{m} E\left\{s_{m}\right\}\right)$ (or $\left.2 \pi r \lambda_{f} E\left\{s_{f}\right\} \exp \left(-\pi r^{2} \lambda_{f} E\left\{s_{f}\right\}\right)\right)$. While in open access mode, the circumstance of MUEs contact with FAP also keep to the above assumptions. The pdf of the distance $R_{m}^{\prime}$ (or $R_{f}^{\prime}$ ) between each MBS (or FAP) and its designated MUE (or FUE) pair is given by $2 \pi r\left(\lambda_{m} E\left\{s_{m}\right\}+\right.$ $\left.\kappa^{2} \lambda_{f} E\left\{s_{f}\right\}\right) \exp \left[-\pi r^{2}\left(\lambda_{m} E\left\{s_{m}\right\}+\kappa^{2} \lambda_{f} E\left\{s_{f}\right\}\right)\right]$ (or $2 \pi r$ $\left[\left(\lambda_{m} / \kappa^{2}\right) E\left\{s_{m}\right\}+\lambda_{f} E\left\{s_{f}\right\}\right] \exp \left[-\pi r^{2}\left(\left(\lambda_{m} / \kappa^{2}\right) E\left\{s_{m}\right\}+\right.\right.$ $\left.\left.\lambda_{f} E\left\{s_{f}\right\}\right)\right]$ ) [14], where $E\left\{s_{m}\right\}=\int_{0}^{1} s_{m}(x) f_{A_{m}}(x) d x$, $E\left\{s_{f}\right\}=\int_{0}^{1} s_{f}(x) f_{A_{f}}(x) d x, f_{A_{m}}(x)$ and $f_{A_{m}}(x)$ are the pdf of $A_{m}$ and $A_{f}$.

\section{Performance evaluation of networks}

In this section, we discuss system performance evaluation in three aspects: downlink coverage probability, power consumption optimization, and energy efficiency.

\subsection{Downlink coverage probability}

When some BSs are turned off, the remaining active BSs will expand coverage to maintain the overall coverage, while the influence of different sleep strategies for coverage are different. Moreover, femtocell access modes have impact on the choice of MUE, which directly influence coverage probability of MUE. Then, coverage probabilities of MUE and FUE with different sleep strategies and femtocell access modes are analyzed in this subsection.

\subsubsection{Random sleeping strategy}

Besides the activity probability of BS, access policy also impacts MUE coverage probability. In addition, the different modes (active/sleep) of MBS would lead different results when femtocell operates in closed access mode. In the following, under the condition of closed access and open access, we assume that there is a centralized control in MBS and investigate users' coverage probability respectively in random sleeping strategy. 


\section{(1) Femtocells operate in closed access mode}

When femtocells operate in closed access mode, the interferers of FUE depend on the mode (active/sleep) of MBSs, so we analyze system performance in two cases: MBSs are all in sleeping mode and MBSs are partly in sleeping mode.

a) MBSs are all in sleeping mode

The coverage probability of a randomly located FUE is given by:

$$
\begin{aligned}
P_{f c, a s}^{f}= & \int_{r=0}^{\infty} \mathcal{L}_{I}(r) \mathcal{L}_{N}(r) f_{R_{f}}(r) d r \\
= & \int_{r=0}^{\infty} \exp \left(-\pi r^{2} \lambda_{f} q_{f}\left(\gamma_{f}\right)^{2 / \alpha} C(\alpha)\right) \\
& \times \exp \left(-\frac{r^{\alpha} \gamma_{f} \sigma^{2}}{Q}\right) 2 \pi \lambda_{f} q_{f} r \exp \left(-\pi \lambda_{f} q_{f} r^{2}\right) d r,
\end{aligned}
$$

where $\mathcal{L}_{I}(r)$ and $\mathcal{L}_{N}(r)$ denote the Laplace transform of interferers and background noise respectively. $\mathcal{L}_{I}(r)$ always be denoted as $\mathcal{L}_{I}(r)=\exp \left(-\pi r^{2} \lambda_{f} q_{f}\right.$ $\left.\left(\frac{P_{I}}{P_{T}} \gamma_{f}\right)^{2 / \alpha} C(\alpha)\right)$ [14], where $P_{T}$ and $P_{I}$ represent the transmitting power of transmitter and interferer. In $(4), \mathcal{L}_{I}(r)=\exp \left(-\pi r^{2} \lambda_{f} q_{f} \gamma_{f}^{2 / \alpha} C(\alpha)\right)[16]^{\mathrm{b}}$ and $C(\alpha)=\frac{2 \pi}{\alpha \sin (2 \pi / \alpha)}$. When MBSs are all in sleeping mode, for $\sigma^{2}=0$, the coverage probability of FUE is $\frac{1}{1+\left(\gamma_{f}\right)^{2 / \alpha} C(\alpha)}$, which is completely independent of the random sleeping policy, and only depends on the parameters $\gamma_{f}$ and $\alpha$.

\section{b) MBSs are partly in sleeping mode}

When MBSs are partly in sleeping mode, different from the above analysis, the interferers contain FAPs and MBSs. The coverage probability of a randomly located MUE is given by:

$$
\begin{aligned}
P_{f c, r s}^{m}= & \int_{r=0}^{\infty} \mathcal{L}_{I}(r) \mathcal{L}_{N}(r) f_{R_{m}}(r) d r \\
= & \int_{r=0}^{\infty} \exp \left(-\pi r^{2} \lambda_{m} q_{m} \rho\left(\gamma_{m}, \alpha\right)\right) \exp \left(-\frac{r^{\alpha} \gamma_{m} \sigma^{2}}{\beta_{m} P}\right) \\
& \times \exp \left(-\pi r^{2} \lambda_{f} q_{f}\left(\gamma_{m} Q / P\right)^{2 / \alpha} C(\alpha)\right) \\
& \times 2 \pi \lambda_{m} q_{m} r \exp \left(-\pi \lambda_{m} q_{m} r^{2}\right) d r,
\end{aligned}
$$

where $\rho\left(\gamma_{m}, \alpha\right)=\gamma_{m}^{2 / \alpha} \int_{\gamma_{m}^{-2 / \alpha}}^{\infty} \frac{1}{1+u^{\alpha / 2}} d u$, and $\mathcal{L}_{I}(r)$ is given by $\exp \left(-\pi r^{2} \lambda_{m} q_{m} \rho\left(\gamma_{m}, \alpha\right)\right)$.
When $\sigma^{2}=0$, (5) can be simplified as:

$$
P_{f c, r s}^{m}=\frac{\lambda_{m} q_{m}}{\lambda_{m} q_{m}\left(\rho\left(\gamma_{m}, \alpha\right)+1\right)+\lambda_{f} q_{f}\left(\gamma_{m} Q / P\right)^{2 / \alpha} C(\alpha)} .
$$

Then, the coverage probability of a randomly located FUE is given by:

$$
\begin{aligned}
P_{f c, r s}^{f}= & \int_{r=0}^{\infty} \mathcal{L}_{I}(r) \mathcal{L}_{N}(r) f_{R_{f}}(r) d r \\
= & \int_{r=0}^{\infty} \exp \left(-\pi r^{2} \lambda_{f} q_{f}\left(\gamma_{f}\right)^{2 / \alpha} C(\alpha)\right) \\
& \times \exp \left(-\pi r^{2} \lambda_{m} q_{m}\left(\gamma_{f} P / Q\right)^{2 / \alpha} C(\alpha)\right) \\
& \times \exp \left(-\frac{r^{\alpha} \gamma_{f} \sigma^{2}}{Q}\right) 2 \pi \lambda_{f} q_{f} r \exp \left(-\pi \lambda_{f} q_{f} r^{2}\right) d r
\end{aligned}
$$

where $\mathcal{L}_{I}(r)$ is denoted as $\exp \left(-\pi r^{2} \lambda_{f} q_{f}\left(\gamma_{f}\right)^{2 / \alpha} C(\alpha)\right) \times$ $\exp \left(-\pi r^{2} \lambda_{m} q_{m}\left(\gamma_{f} P / Q\right)^{2 / \alpha} C(\alpha)\right)$.

When $\sigma^{2}=0$, (7) can be simplified as:

$$
P_{f c, r s}^{f}=\frac{\lambda_{f} q_{f}}{\lambda_{f} q_{f}\left(\gamma_{f}\right)^{2 / \alpha} C(\alpha)+\lambda_{m} q_{m}\left(\gamma_{f} P / Q\right)^{2 / \alpha} C(\alpha)+\lambda_{f} q_{f}} .
$$

From (8), we find that the coverage probability of FUE depends on the activity probability of FAP. However, when the intensity of MBSs $\lambda_{m}$ is far less than the intensity of FAPs $\lambda_{f}$, (8) can be simplified to $\frac{1}{1+\left(\gamma_{f}\right)^{2 / \alpha} C(\alpha)}$, so we get that FUE coverage probability does not change with $q_{m}$ and $q_{f}$ when $\lambda_{f} \gg \lambda_{m}$, then we can ignore the impact.

\section{(2) Femtocells operate in open access mode}

When femtocells operate in open access mode, MUEs will have chance to access FAPs. The coverage probability of a randomly located MUE can be obtained in the following theorem.

Theorem 1. When femtocells operate in open access mode, and MBS and FAP are both in random sleeping strategy, the coverage probability of a randomly located MUE is given by:

$$
\begin{aligned}
P_{f o, r s}^{m}= & \int_{r=0}^{\infty} \mathcal{L}_{I \mid M}(r) \mathcal{L}_{N \mid M}(r) f_{R_{m} \mid M}(r) d r \times \frac{q_{m} \lambda_{m}}{\kappa^{2} \lambda_{f} q_{f}+q_{m} \lambda_{m}} \\
& +\int_{r=0}^{\infty} \mathcal{L}_{I \mid F}(r) \mathcal{L}_{N \mid F}(r) f_{R_{f} \mid F}(r) d r \times \frac{\kappa^{2} q_{f} \lambda_{f}}{\kappa^{2} \lambda_{f} q_{f}+q_{m} \lambda_{m}},
\end{aligned}
$$

where $\mathcal{L}_{I \mid M}(r)=\exp \left(-\pi r^{2} \kappa^{2} q_{f} \lambda_{f} \rho\left(\frac{\gamma_{m} Q}{\kappa^{\alpha} P}, \alpha\right)\right) \times \exp (-\pi$ $\left.r^{2} q_{m} \lambda_{m} \rho\left(\gamma_{m}, \alpha\right)\right), \mathcal{L}_{N \mid M}(r)=\exp \left(\frac{r^{\alpha} \gamma_{m} \sigma^{2}}{\beta_{m} P}\right), \mathcal{L}_{I \mid F}(r)=$ 
$\exp \left(-\pi\left(\frac{r}{\kappa}\right)^{2} q_{m} \lambda_{m} \rho\left(\frac{\gamma_{f} P^{\alpha}}{Q}, \alpha\right)\right) \times \exp \left(-\pi r^{2} q_{f} \lambda_{f} \rho\right.$ $\left.\left(\gamma_{f}, \alpha\right)\right), \mathcal{L}_{N \mid F}(r)=\exp \left(\frac{r^{\alpha} \gamma_{f} \sigma^{2}}{Q}\right), f_{R_{m} \mid M}(r)=2 \pi r$ $\left(q_{m} \lambda_{m}+q_{f} \lambda_{f} \kappa^{2}\right) \exp \left(-\pi r^{2}\left(q_{m} \lambda_{m}+q_{f} \lambda_{f} \kappa^{2}\right)\right), f_{R_{f} \mid F}(r)=$ $2 \pi r\left(\frac{q_{m} \lambda_{m}}{\kappa^{2}}+q_{f} \lambda_{f}\right) \exp \left(-\pi r^{2}\left(\frac{q_{m} \lambda_{m}}{\kappa^{2}}+q_{f} \lambda_{f}\right)\right)$.

Proof. See Appendix 1.

When $\sigma^{2}=0$, (9) can be simplified as:

$$
\begin{aligned}
P_{f o, r s}^{m}= & \frac{\lambda_{m} q_{m}}{\left(\lambda_{m} q_{m}+\kappa^{2} \lambda_{f} q_{f}\right) \times\left[\rho\left(\gamma_{m}, \alpha\right)+1\right]} \\
& +\frac{\lambda_{f} q_{f} \kappa^{2}}{\left(\lambda_{m} q_{m}+\kappa^{2} \lambda_{f} q_{f}\right) \times\left[\rho\left(\gamma_{f}, \alpha\right)+1\right]}
\end{aligned}
$$

It is worth noting that when femtocells operate in open access mode, the coverage probability of FUE is same with what is in the circumstance that femtocells operate in closed access and MBSs are partly in sleeping mode.

In subsequent sections, to get closed form expressions simplified, we will take $\kappa=(Q / P)^{1 / \alpha}[17,18]$.

\subsubsection{Activity-aware sleeping strategy}

(1) Femtocells operate in closed access mode

The coverage probability of the active MUE $P_{f c, p s}^{m}$ with the proposed strategy and closed access mode is given in (11):

$$
\begin{aligned}
P_{f, p s}^{m}= & \frac{1}{E\left\{a_{m}\right\}} \int_{0}^{1} x P\left(\operatorname{SINR}>\gamma_{m} \mid x\right) f_{A_{m}}(x) d x \\
= & \frac{1}{E\left\{a_{m}\right\}}\left\{\int _ { 0 } ^ { 1 } \left[x P\left(N_{m}=1\right) P\left(\operatorname{SINR}>\gamma_{m} \mid N_{m}=1\right)\right.\right. \\
& \left.\left.+x P\left(N_{m}>1\right) P\left(\operatorname{SINR}>\gamma_{m} \mid N_{m}>1\right)\right] f_{A_{m}}(x) d x\right\} \\
= & \frac{1}{E\left\{a_{m}\right\}}\left\{\int _ { 0 } ^ { 1 } \left[x s_{m}(x) P\left(\operatorname{SINR}>\gamma_{m} \mid N_{m}=1\right)\right.\right. \\
& \left.\left.+x\left(1-s_{m}(x)\right) P\left(\operatorname{SINR}>\gamma_{m} \mid N_{m}>1\right)\right] f_{A_{m}}(x) d x\right\} \\
= & \frac{1}{E\left\{a_{m}\right\}} \int_{0}^{1} x s_{m}(x) f_{A_{m}}(x) d x \int_{r=0}^{\infty} 2 \pi r \lambda_{m} \\
& \times \exp \left(-\pi r^{2} \lambda_{m}\left(E\left\{s_{m}\right\} \rho\left(\gamma_{m}, \alpha\right)+1\right)\right) \exp \left(-\pi r^{2} \lambda_{f} E\left\{s_{f}\right\}\right. \\
& \left.\times\left(\frac{\gamma_{m} Q}{P}\right)^{\frac{2}{\alpha}} C(\alpha)\right) \exp \left(\frac{-r^{\alpha} \gamma_{m} \sigma^{2}}{\beta_{m} P}\right) d r+\frac{1}{E\left\{a_{m}\right\}} \\
& \int_{0}^{1} x\left(1-s_{m}(x)\right) f_{A_{m}}(x) d x \sum_{i=2}^{\infty} E\left\{s_{m}\right\}\left(1-E\left\{s_{m}\right\}\right)^{i-2} \\
& \int_{r=0}^{\infty} \exp \left(-\pi r^{2} \lambda_{m}\left(E\left\{s_{m}\right\} \rho\left(\gamma_{m}, \alpha\right)+1\right)\right) \\
& \times \exp \left(-\pi r^{2} \lambda_{f} E\left\{s_{f}\right\}\left(\frac{\gamma_{m} Q}{P}\right)^{\frac{2}{\alpha}} C(\alpha)\right) \\
& \times \exp \left(\frac{-r^{\alpha} \gamma_{m} \sigma^{2}}{\beta_{m} P}\right) 2\left(\pi \lambda_{m}\right)^{i} r^{2 i-1} d r, \\
& x(11)
\end{aligned}
$$

where $P\left(\operatorname{SINR}>\gamma_{m} \mid N_{m}>1\right)$ can be taken as:

$$
\begin{aligned}
\sum_{i=2}^{\infty} & \frac{E\left\{s_{m}\right\}\left(1-E\left\{s_{m}\right\}\right)^{i-2}\left(\lambda_{m}\right)^{i}}{\left[\lambda_{m}\left(E\left\{s_{m}\right\} \rho_{m}+1\right)+\lambda_{f} E\left\{s_{f}\right\}\left(\frac{\gamma_{m} Q}{P}\right)^{2 / \alpha} C(\alpha)\right]^{i}} \\
= & \frac{\lambda_{m}^{2} E\left\{s_{m}\right\}}{\lambda_{m}\left(E\left\{s_{m}\right\} \rho_{m}+1\right)+\lambda_{f} E\left\{s_{f}\right\}\left(\frac{\gamma_{m} Q}{P}\right)^{2 / \alpha} C(\alpha)} \\
& \times \frac{1}{\lambda_{m} E\left\{s_{m}\right\}\left(\rho_{m}+1\right)+\lambda_{f} E\left\{s_{f}\right\}\left(\frac{\gamma_{m} Q}{P}\right)^{2 / \alpha} C(\alpha)}
\end{aligned}
$$

When $\sigma^{2}=0,(11)$ is taken as:

$$
\begin{aligned}
P_{f c, p s}^{m}= & \frac{\lambda_{m} E\left\{a_{m} s_{m}\left(a_{m}\right)\right\} / E\left\{a_{m}\right\}}{\lambda_{m}\left[E\left\{s_{m}\right\} \rho\left(\gamma_{m}, \alpha\right)+1\right]+\lambda_{f} E\left\{s_{f}\right\}\left(\frac{\gamma_{m} Q}{P}\right)^{\frac{2}{\alpha}} C(\alpha)} \\
& +\frac{\left[E\left\{a_{m}\right\}-E\left\{a_{m} s_{m}\left(a_{m}\right)\right\}\right] / E\left\{a_{m}\right\}}{\lambda_{m}\left[E\left\{s_{m}\right\} \rho\left(\gamma_{m}, \alpha\right)+1\right]+\lambda_{f} E\left\{s_{f}\right\}\left(\frac{\gamma_{m} Q}{P}\right)^{\frac{2}{\alpha}} C(\alpha)} \\
& \times \frac{\lambda_{m}^{2} E\left\{s_{m}\right\}}{\lambda_{m} E\left\{s_{m}\right\}\left[\rho\left(\gamma_{m}, \alpha\right)+1\right]+\lambda_{f} E\left\{s_{f}\right\}\left(\frac{\gamma_{m} Q}{P}\right)^{\frac{2}{\alpha}} C(\alpha)} .
\end{aligned}
$$

Lemma 1. When $\sigma^{2}=0$ and $\lambda_{f} \gg \lambda_{m}$, if satisfying the following inequality constraints (14), the proposed sleeping strategy can improve the coverage probability of the active MUE compared with the corresponding random policy with closed access mode.

$$
E\left\{a_{m} s_{m}\left(a_{m}\right)\right\}>E\left\{a_{m}\right\} E\left\{s_{m}\right\} .
$$

Proof. The proof is only based on the standard algebraic manipulations and the condition of $\lambda_{f} \gg \lambda_{m}$, so it is omitted here.

Being similar with (13), the coverage probability of the active FUE is given by:

$$
\begin{aligned}
P_{f c, p s}^{f}= & \frac{\lambda_{f} E\left\{a_{f} s_{f}\left(a_{f}\right)\right\} / E\left\{a_{f}\right\}}{\lambda_{f}\left[E\left\{s_{f}\right\} \rho\left(\gamma_{f}, \alpha\right)+1\right]+\lambda_{m} E\left\{s_{m}\right\}\left(\frac{\gamma_{f} Q}{P}\right)^{\frac{2}{\alpha}} C(\alpha)} \\
& +\frac{\left[E\left\{a_{f}\right\}-E\left\{a_{f} s_{f}\left(a_{f}\right)\right\}\right] / E\left[a_{f}\right]}{\lambda_{f}\left[E\left\{s_{f}\right\} \rho\left(\gamma_{f}, \alpha\right)+1\right]+\lambda_{m} E\left\{s_{m}\right\}\left(\frac{\gamma_{f} P}{Q}\right)^{\frac{2}{\alpha}} C(\alpha)} \\
& \times \frac{\lambda_{f}^{2} E\left\{s_{f}\right\}}{\lambda_{f} E\left\{s_{f}\right\}\left[\rho\left(\gamma_{f}, \alpha\right)+1\right]+\lambda_{m} E\left\{s_{m}\right\}\left(\frac{\gamma_{f} P}{Q}\right)^{\frac{2}{\alpha}} C(\alpha)} .
\end{aligned}
$$


(2) Femtocells operate in open access mode

Based on the strategy proposed in Section 4.2, we can reach the coverage probability of the active MUE in the following theorem.

Theorem 2. When $\sigma^{2}=0$, the coverage probability of the active MUE $P_{f o, p s}^{m}$ with the proposed strategy and open access mode is shown in (16):

$$
\begin{aligned}
P_{f o, p s}^{m}= & \left\{\frac{E\left\{a_{m} s_{m}\left(a_{m}\right)\right\}}{E\left\{a_{m}\right\}} \times \frac{\lambda_{m}+\kappa^{2} \lambda_{f}}{\lambda_{m}+\kappa^{2} \lambda_{f}+\rho\left(\gamma_{m}, \alpha\right) H(m, f)}\right. \\
& +\frac{E\left\{s_{m}\right\}\left(\lambda_{m}+\kappa^{2} \lambda_{f}\right)^{2} / E\left\{a_{m}\right\}}{\lambda_{m}+\kappa^{2} \lambda_{f}+\rho\left(\gamma_{m}, \alpha\right) H(m, f)} \\
& \left.\times \frac{E\left\{a_{m}\right\}-E\left\{a_{m} s_{m}\left(a_{m}\right)\right\}}{J(m)+\kappa^{2} \lambda_{f}\left[E\left\{s_{f}\right\} \rho\left(\gamma_{m}, \alpha\right)+E\left\{s_{m}\right\}\right]}\right\} \\
& \times P(M)+\left\{\frac{E\left\{a_{m} s_{f}\left(a_{m}\right)\right\}}{E\left\{a_{m}\right\}} \times \frac{\lambda_{m} / \kappa^{2}+\lambda_{f}}{\lambda_{m} / \kappa^{2}+\lambda_{f}+\rho\left(\gamma_{f}, \alpha\right) I(m, f)}\right. \\
& +\frac{E\left\{a_{m}\right\}-E\left\{a_{m} s_{f}\left(a_{m}\right)\right\}}{\left(\lambda_{m} / \kappa^{2}\right)\left[E\left\{s_{m}\right\} \rho\left(\gamma_{f}, \alpha\right)+E\left\{s_{f}\right\}\right]+J(f)} \\
& \left.\times \frac{E\left\{s_{f}\right\}\left(\lambda_{m} / \kappa^{2}+\lambda_{f}\right)^{2} / E\left\{a_{m}\right\}}{\lambda_{m} / \kappa^{2}+\lambda_{f}+\rho\left(\gamma_{f}, \alpha\right) I(m, f)}\right\} \times P(F),
\end{aligned}
$$

where $J(m)=\lambda_{m} E\left\{s_{m}\right\}\left[\rho\left(\gamma_{m}, \alpha\right)+1\right], J(f)=\lambda_{f} E\left\{s_{f}\right\}$ $\left[\rho\left(\gamma_{f}, \alpha\right)+1\right], H(m, f)=\lambda_{m} E\left\{s_{m}\right\}+\kappa^{2} \lambda_{f} E\left\{s_{f}\right\}, I(m, f)=$ $\left(\lambda_{m} / \kappa^{2}\right) E\left\{s_{m}\right\}+\lambda_{f} E\left\{s_{f}\right\}, P(M)=\frac{E\left\{s_{m}\right\} \lambda_{m}}{\kappa^{2} \lambda_{f} E\left\{s_{f}\right\}+E\left\{s_{m}\right\} \lambda_{m}}$, $P(F)=\frac{\lambda_{f} E\left\{s_{f}\right\} \kappa^{2}}{\lambda_{f} E\left\{s_{f}\right\} \kappa^{2}+\lambda_{m} E\left\{s_{m}\right\}}$.

\section{Proof. See Appendix 2.}

The coverage probability of active FUE affected by the activity activity of BS and UE is the same with (15).

\subsection{Power consumption optimization}

We formulate optimization problems in the form of power consumption minimization to determine the optimal operating frequency of the MBSs and FAPs.

\subsubsection{Random sleeping strategy}

The power consumption minimization problem in random sleeping strategy with femtocell closed access mode is formulated as:

$$
\begin{array}{ll}
\min _{q_{m}, q_{f}} & P_{m, r s}+P_{f, r s} \\
\text { s.t. } \quad & P_{f c, r s}^{m} \geq \varepsilon_{m r} \\
& P_{f c, r s}^{f} \geq \varepsilon_{f r}
\end{array}
$$

where $P_{m, r s}$ and $P_{f, r s}$ represent the power consumption of MBSs and FAPs in random sleeping strategy, respectively, which are denoted by $P_{m, r s}=\lambda_{m} q_{m}\left(P_{m 0}+\Delta M \beta_{m} P_{m}\right)+$ $\lambda_{m}\left(1-q_{m}\right) P_{m s l e e p}$ and $P_{f, r s}=\lambda_{f} q_{f}\left(P_{f 0}+\Delta f P_{f}\right)+\lambda_{f}(1-$ $\left.q_{f}\right) P_{\text {fsleep }} . P_{\text {msleep }}$ and $P_{\text {fsleep }}$ are the power consumed by a MBS and FAP in sleeping mode, respectively. In (17a) and (17b), $\varepsilon_{m r}$ and $\varepsilon_{f r}$ are the constraints of MUE and FUE coverage probability, respectively, which can be taken as the network coverage and users' QoS constraints.

To solve the above problem (17), using Lemma 3 in [11], we would find that (17a) and (17b) are increasing functions of $q_{m}$ and $q_{f}$, respectively. Then, we conclude that $q_{m}^{*}$ and $q_{f}^{*}$ satisfy the constraints tightly and reach the minimum total power consumption simultaneously.

So $q_{m}^{*}$ and $q_{f}^{*}$ can be taken as:

$$
\begin{aligned}
\varepsilon_{m r}= & \int_{r=0}^{\infty} \exp \left(-\pi r^{2} \lambda_{m} q_{m}^{*}\left(\rho\left(\gamma_{m}, \alpha\right)+1\right)\right) \exp \left(-\frac{r^{\alpha} \gamma_{m} \sigma^{2}}{\beta_{m} P}\right) \\
& \times \exp \left(-\pi r^{2} \lambda_{f} q_{f}^{*}\left(\frac{\gamma_{m} Q}{P}\right)^{2 / \alpha} C(\alpha)\right) 2 \pi \lambda_{m} q_{m}^{*} r d r \\
\varepsilon_{f r}= & \int_{r=0}^{\infty} \exp \left(-\pi r^{2} \lambda_{m} q_{m}^{*}\left(\frac{\gamma_{f} P}{Q}\right)^{2 / \alpha} C(\alpha)\right) \exp \left(-\frac{r^{\alpha} \gamma_{f} \sigma^{2}}{Q}\right) \\
& \times \exp \left(-\pi r^{2} \lambda_{f} q_{f}^{*}\left(\gamma_{f}^{2 / \alpha} C(\alpha)+1\right)\right) 2 \pi \lambda_{f} q_{f}^{*} r d r .
\end{aligned}
$$

When $\sigma^{2}=0$, (18) can be simplified as:

$$
\begin{aligned}
\varepsilon_{m r} & =\frac{\lambda_{m} q_{m}^{*}}{\lambda_{m} q_{m}^{*}\left[\rho\left(\gamma_{m}, \alpha\right)+1\right]+\lambda_{f} q_{f}^{*}\left(\frac{\gamma_{m} Q}{P}\right)^{2 / \alpha} C(\alpha)}, \\
\varepsilon_{f r} & =\frac{\lambda_{f} q_{f}^{*}}{\lambda_{f} q_{f}^{*}\left[\gamma_{f}^{2 / \alpha} C(\alpha)+1\right]+\lambda_{m} q_{m}^{*}\left(\frac{\gamma_{f} P}{Q}\right)^{2 / \alpha} C(\alpha)}
\end{aligned}
$$

When femtocell operates in open access mode, the coverage probability of FUE almost has no change, so we only analyze the coverage probability of MUE. Similar with the minimization problem (17), the optimum value of $q_{m}^{\prime *}$ which can reach the total power consumption satisfy:

$$
\begin{aligned}
P_{f o, r s}^{m}= & \int_{r=0}^{\infty} \mathcal{L}_{I \mid M}(r) \mathcal{L}_{N \mid M}(r) f_{R_{m} \mid M}(r) d r \times \frac{q_{m}^{\prime *} \lambda_{m}}{\kappa^{2} \lambda_{f} q_{f}^{\prime *}+q_{m}^{\prime *} \lambda_{m}} \\
& +\int_{r=0}^{\infty} \mathcal{L}_{I \mid F}(r) \mathcal{L}_{N \mid F}(r) f_{R_{f} \mid F}(r) d r \times \frac{\kappa^{2} q_{f}^{\prime *} \lambda_{f}}{\kappa^{2} \lambda_{f} q_{f}^{\prime *}+q_{m}^{\prime *} \lambda_{m}} .
\end{aligned}
$$


When $\sigma^{2}=0$, (20) can be taken as:

$$
\begin{aligned}
\varepsilon_{m r}^{\prime}= & \frac{\lambda_{m} q_{m}^{\prime *}}{\left(\lambda_{m} q_{m}^{\prime *}+\kappa^{2} \lambda_{f} q_{f}^{\prime *}\right) \times\left[\rho\left(\gamma_{m}, \alpha\right)+1\right]} \\
& +\frac{\lambda_{f} q_{f}^{\prime *} \kappa^{2}}{\left(\lambda_{m} q_{m}^{\prime *}+\kappa^{2} \lambda_{f} q_{f}^{\prime *}\right) \times\left[\rho\left(\gamma_{f}, \alpha\right)+1\right]} .
\end{aligned}
$$

\subsubsection{Activity-aware sleeping strategy}

When femtocell operates in closed access mode, the power consumption minimization problem with the proposed sleeping strategy is expressed as:

$$
\begin{array}{lc}
\min _{E\left\{s_{m}\right\}, E\left\{s_{f}\right\}} & P_{m, p s 1}+P_{f, p s 1} \\
\text { s.t. } \quad & P_{f c, p s}^{m} \geq \varepsilon_{m p} \\
& P_{f c, p s}^{f} \geq \varepsilon_{f p}
\end{array}
$$

where $P_{m, p s 1}=\lambda_{m} E\left\{s_{m}\right\}\left(P_{m 0}+\Delta M \beta_{m} P_{m}\right)+\lambda_{m}(1-$ $\left.E\left\{s_{m}\right\}\right) P_{\text {msleep }}, P_{f, p s 1}=\lambda_{f} E\left\{s_{f}\right\}\left(P_{f 0}+\Delta f P_{f}\right)+\lambda_{f}(1-$ $\left.E\left\{s_{f}\right\}\right) P_{\text {fsleep }}$. Constraints (22a) and (22b) are also can be taken as the network coverage and users' QoS constraints. The strategy mainly depends on the activity level of BS, then we can solve this minimization problem (22) through activity level. Marking threshold $a_{m}$ and $a_{f}$ as $a_{0}$ in Lemma 4 [11], we assume that MBS is active when its activity level exceed $a_{m}$, and FAP is active when its activity level exceed $a_{f}$. Similarly to Theorem 3 [11], optimal $a_{m}^{*}$ and $a_{f}^{*}$ satisfy:

$$
\begin{aligned}
\varepsilon_{m p}= & \frac{1}{E\left\{a_{m}\right\}} P\left(\operatorname{SINR}>\gamma_{m} \mid N_{m}=1\right) \int_{a_{m}^{*}}^{1} x f_{A_{m}}(x) d x \\
& +\frac{1}{E\left\{a_{m}\right\}} P\left(\operatorname{SINR}>\gamma_{m} \mid N_{m}>1\right) \int_{0}^{a_{m}^{*}} x f_{A_{m}}(x) d x \\
\varepsilon_{f p}= & \frac{1}{E\left\{a_{f}\right\}} P\left(\operatorname{SINR}>\gamma_{f} \mid N_{f}=1\right) \int_{a_{f}^{*}}^{1} x f_{A_{f}}(x) d x \\
& +\frac{1}{E\left\{a_{f}\right\}} P\left(\operatorname{SINR}>\gamma_{f} \mid N_{f}>1\right) \int_{0}^{a_{f}^{*}} x f_{A_{f}}(x) d x .
\end{aligned}
$$

When $\sigma^{2}=0$, (23) can be expressed by:

$$
\begin{aligned}
\varepsilon_{m p}= & \frac{\lambda_{m} E\left\{a_{m}^{*} s_{m}\left(a_{m}^{*}\right)\right\} / E\left\{a_{m}\right\}}{\lambda_{m}\left[E\left\{s_{m}\right\} \rho\left(\gamma_{m}, \alpha\right)+1\right]+\lambda_{f} E\left\{s_{f}\right\}\left(\frac{\gamma_{m} Q}{P}\right)^{\frac{2}{\alpha}} C(\alpha)} \\
& +\frac{\left[E\left\{a_{m}^{*}\right\}-E\left\{a_{m}^{*} s_{m}\left(a_{m}^{*}\right)\right\}\right] / E\left\{a_{m}\right\}}{\lambda_{m}\left[E\left\{s_{m}\right\} \rho\left(\gamma_{m}, \alpha\right)+1\right]+\lambda_{f} E\left\{s_{f}\right\}\left(\frac{\gamma_{m} Q}{P}\right)^{\frac{2}{\alpha}} C(\alpha)} \\
& \times \frac{\lambda_{m}^{2} E\left\{s_{m}\right\}}{\lambda_{m} E\left\{s_{m}\right\}\left[\rho\left(\gamma_{m}, \alpha\right)+1\right]+\lambda_{f} E\left\{s_{f}\right\}\left(\frac{\gamma_{m} Q}{P}\right)^{\frac{2}{\alpha}} C(\alpha)}
\end{aligned}
$$

$$
\begin{aligned}
\varepsilon_{f p}= & \frac{\lambda_{f} E\left\{a_{f}^{*} s_{f}\left(a_{f}^{*}\right)\right\} / E\left\{a_{f}\right\}}{\lambda_{f}\left[E\left\{s_{f}\right\} \rho\left(\gamma_{f}, \alpha\right)+1\right]+\lambda_{m} E\left\{s_{m}\right\}\left(\frac{\gamma_{f} P}{Q}\right)^{\frac{2}{\alpha}} C(\alpha)} \\
& +\frac{\left[E\left\{a_{f}^{*}\right\}-E\left\{a_{f}^{*} s_{f}\left(a_{f}^{*}\right)\right\}\right] / E\left\{a_{f}\right\}}{\lambda_{f}\left[E\left\{s_{f}\right\} \rho\left(\gamma_{f}, \alpha\right)+1\right]+\lambda_{m} E\left\{s_{m}\right\}\left(\frac{\gamma_{f} P}{Q}\right)^{\frac{2}{\alpha}} C(\alpha)} \\
& \times \frac{\lambda_{f}^{2} E\left\{s_{f}\right\}}{\lambda_{f} E\left\{s_{f}\right\}\left[\rho\left(\gamma_{f}, \alpha\right)+1\right]+\lambda_{m} E\left\{s_{m}\right\}\left(\frac{\gamma_{f} P}{Q}\right)^{\frac{2}{\alpha}} C(\alpha)} .
\end{aligned}
$$

When femtocell operates in open access mode, similarly to the status of optimization problems for random strategy, only the coverage probability of MUE is analyzed. Then optimal $a_{m}^{\prime *}$ satisfies the following expression:

$$
\begin{aligned}
\varepsilon_{m p}^{\prime}= & \frac{1}{E\left\{a_{m}^{\prime}\right\}} P\left(\operatorname{SINR}>\gamma_{m} \mid N_{m}=1\right) \int_{a_{m}^{\prime *}}^{1} x f_{A_{m}^{\prime}}(x) d x \\
& \times P(M)+\frac{1}{E\left\{a_{m}^{\prime}\right\}} P\left(\operatorname{SINR}>\gamma_{m} \mid N_{m}>1\right) \\
& \int_{0}^{a_{m}^{\prime *}} x f_{A_{m}^{\prime}}(x) d x \times P(M) .
\end{aligned}
$$

When $\sigma^{2}=0$, we also can obtain the closed expression of (25) in (26):

$$
\begin{aligned}
\varepsilon_{m p}^{\prime}= & \left\{\frac{E\left\{a_{m}^{\prime *} s_{m}\left(a_{m}^{\prime *}\right)\right\}}{E\left\{a_{m}^{\prime}\right\}} \times \frac{\lambda_{m}+\kappa^{2} \lambda_{f}}{\lambda_{m}+\kappa^{2} \lambda_{f}+\rho\left(\gamma_{m}, \alpha\right) H(m, f)}\right. \\
& +\frac{E\left\{s_{m}\right\}\left(\lambda_{m}+\kappa^{2} \lambda_{f}\right)^{2} / E\left\{a_{m}^{\prime}\right\}}{\lambda_{m}+\kappa^{2} \lambda_{f}+\rho\left(\gamma_{m}, \alpha\right) H(m, f)} \\
& \left.\times \frac{E\left\{a_{m}^{\prime *}\right\}-E\left\{a_{m}^{\prime *} s_{m}\left(a_{m}^{\prime *}\right)\right\}}{J(m)+\kappa^{2} \lambda_{f}\left[E\left\{s_{f}\right\} \rho\left(\gamma_{m}, \alpha\right)+E\left\{s_{m}\right\}\right]}\right\} \\
& \times P(M)+\left\{\frac{E\left\{a_{m}^{\prime *} s_{f}\left(a_{m}^{\prime *}\right)\right\}}{E\left\{a_{m}^{\prime}\right\}} \times \frac{\lambda_{m} / \kappa^{2}+\lambda_{f}}{\lambda_{m} / \kappa^{2}+\lambda_{f}+\rho\left(\gamma_{f}, \alpha\right) I(m, f)}\right. \\
& +\frac{E\left\{a_{m}^{\prime *}\right\}-E\left\{a_{m}^{\prime *} s_{f}\left(a_{m}^{\prime *}\right)\right\}}{\left(\lambda_{m} / \kappa^{2}\right)\left[E\left\{s_{m}\right\} \rho\left(\gamma_{f}, \alpha\right)+E\left\{s_{f}\right\}\right]+J(f)} \\
& \left.\times \frac{E\left\{s_{f}\right\}\left(\lambda_{m} / \kappa^{2}+\lambda_{f}\right)^{2} / E\left\{a_{m}^{\prime}\right\}}{\lambda_{m} / \kappa^{2}+\lambda_{f}+\rho\left(\gamma_{f}, \alpha\right) I(m, f)}\right\} \times P(F) .
\end{aligned}
$$

\subsection{Energy efficiency}

Besides the problems of coverage probability and power consumption, we also investigate energy efficiency issue for the two-tier femtocell network. The macrocell and femtocell throughput are denoted as $T_{m}$ and $T_{f}$, respectively, which are defined in Section 3. In addition, we 
define $T_{m, c}=\lambda_{m} P_{m, c} \log _{2}\left(1+\gamma_{m}\right)$ and $T_{m, o}=\lambda_{m} P_{m, o}$ $\log _{2}\left(1+\gamma_{m}\right)$ as the expressions for macrocell throughput with closed and open access modes, respectively; then the expressions for total throughput with closed and open access modes are given by $T_{\text {tot }, c}=T_{m, c}+T_{f}$ and $T_{\text {tot }, o}=$ $T_{m, o}+T_{f}$ [17]. Expressions for macrocell throughput with different femtocell access modes and sleeping strategies are expressed by $T_{f c, r s}^{m}=\lambda_{m} P_{f c, r s}^{m} \log _{2}\left(1+\gamma_{m}\right), T_{f o, r s}^{m}=$ $\lambda_{m} P_{f o, r s}^{m} \log _{2}\left(1+\gamma_{m}\right), T_{f c, p s}^{m}=\lambda_{m} P_{f c, p s}^{m} \log _{2}\left(1+\gamma_{m}\right)$, $T_{f o, p s}^{m}=\lambda_{m} P_{f o, p s}^{m} \log _{2}\left(1+\gamma_{m}\right)$.

In the following, energy efficiency is discussed with different sleeping strategies and femtocell access policies.

\subsubsection{Random sleeping strategy}

When femtocells work in closed access mode, the network energy efficient is given by:

$$
\begin{aligned}
\eta_{f c, r s} & =\frac{T_{f c, r s}^{m}+T_{f}}{P_{m, r s}+P_{f, r s}} \\
& =\frac{\lambda_{m} P_{f c, r s}^{m} \log _{2}\left(1+\gamma_{m}\right)+\lambda_{f} P_{f c, r s}^{f} \log _{2}\left(1+\gamma_{f}\right)}{P_{m, r s}+P_{f, r s}} .
\end{aligned}
$$

when femtocells work in open access mode, the network energy efficient is given by:

$$
\begin{aligned}
\eta_{f o, r s} & =\frac{T_{f o, r s}^{m}+T_{f}}{P_{m, r s}+P_{f, r s}} \\
& =\frac{\lambda_{m} P_{f o, r s}^{m} \log _{2}\left(1+\gamma_{m}\right)+\lambda_{f} P_{f o, r s}^{f} \log _{2}\left(1+\gamma_{f}\right)}{P_{m, r s}+P_{f, r s}} .
\end{aligned}
$$

\subsubsection{Activity-aware sleeping strategy}

When femtocells operate in closed access mode, the network energy efficient is given by:

$$
\begin{aligned}
\eta_{f c, p s} & =\frac{T_{f c, p s}^{m}+T_{f}}{P_{m, p s}+P_{f, p s}} \\
& =\frac{\lambda_{m} P_{f c, p s}^{m} \log _{2}\left(1+\gamma_{m}\right)+\lambda_{f} P_{f c, p s}^{f} \log _{2}\left(1+\gamma_{f}\right)}{P_{m, p s}+P_{f, p s}} .
\end{aligned}
$$

When femtocells operate in open access mode, the network energy efficient is given by:

$$
\begin{aligned}
\eta_{f o, p s} & =\frac{T_{f o, p s}^{m}+T_{f}}{P_{m, p s}+P_{f, p s}} \\
& =\frac{\lambda_{m} P_{f o, p s}^{m} \log _{2}\left(1+\gamma_{m}\right)+\lambda_{f} P_{f o, p s}^{f} \log _{2}\left(1+\gamma_{f}\right)}{P_{m, p s}+P_{f, p s}} .
\end{aligned}
$$

\section{Numerical results}

In this section, simulation results are firstly compared to the analytical derivations. We adopt the Monte Carlo method with sufficient number of iterations. For convenience, the proposed sleeping strategy with closed access modes (CAM) for femtocell and open access modes (OAM) for femtocell are abbreviated as PS (CAM) and PS (OAM) and strategic sleeping strategy with CAM for femtocell proposed in [11], which is abbreviated as SS (CAM). For comparison, random sleeping strategy with CAM for femtocell and OAM for femtocell are denoted as RS (CAM) and RS (OAM), respectively. In the following, we use these default values in Table 1, unless otherwise stated.

We have a total of 5,000 trials in the Monte Carlo computation. At each trial, the locations of MBSs and FAPs are distributed according to a PPP in a $1 \mathrm{~km} \times 1 \mathrm{~km}$ square area, respectively, and each user is assumed to connect to the BS with the largest channel gain. Each BS selects user according to the user's state (active/sleep) and distance between BS and user. In addition, additive noise is considered in the simulations.

Figure 3 shows the comparison between the analytical results and simulated results. It is easy to find that the curves of simulated data are fitted highly with the analytical data. We verify the validity of the new expression (16) with different values of $\gamma_{m}$ and $\gamma_{f}\left(\gamma_{f}=2,4,7\right)$ for $\sigma^{2}=0$.

Figure 4 show the coverage probabilities for different noise $\sigma^{2}$ and $\beta_{m}$ with different sleeping strategies and femtocell access modes.

To analyze the effects of noise, we take the following statements and assumptions: for random sleeping strategy, BS stays awake with probability 0.5 and sleeps with probability 0.5 . To verify the feasibility of our strategies, we assume that in the strategic and the proposed sleeping strategy, the activity level of BS is defined as 0 and 1, and the sleeping strategy is 0 and 1 with equal probability 0.5, respectively. According to this activity level model, we can obtain that when $\lambda_{f} \gg \lambda_{m}$, (15) can be simplified to $\frac{E\left\{a_{f} s_{f}\left(a_{f}\right)\right\}}{E\left\{a_{f}\right\}}$, which is not relevant to $E\left\{s_{f}\left(a_{f}\right)\right\}$ and $E\left\{s_{m}\left(a_{m}\right)\right\}$, so we omit the analysis on the coverage probability of FUE.

From the comparison of Figure 4a, we can see that the coverage probability in different strategies decreases with $\sigma^{2}$, while is hardly affected by the coefficient of power control $\beta_{m}$. In addition, the proposed sleeping strategy can increase coverage probability of MUEs obviously in Figure $4 b$, which is caused by the policy of sleeping strategy taking activity level into consideration. Moreover, the curves of MUE coverage probabilities with open access mode for femtocell are all kept upon the curves of closed access mode in different sleeping strategies, which is consistent with the mechanism of open access mode for femtocell. 
Table 1 Notations and parameters

\begin{tabular}{|c|c|c|}
\hline Symbol & Meaning of symbol & Value \\
\hline$\overline{\lambda_{m}}$ & Intensity of MBSs & $1 \times 10^{-4} \mathrm{~m}^{-2}$ \\
\hline$\lambda_{f}$ & Intensity of FAPs & $1 \times 10^{-2} \mathrm{~m}^{-2}$ \\
\hline$\sigma^{2}$ & $\begin{array}{l}\text { Variance of additive white } \\
\text { Gaussian noise }\end{array}$ & 1 \\
\hline$\alpha$ & Path loss exponent & 4 \\
\hline$\beta_{m}$ & $\begin{array}{l}\text { Coefficient of power control } \\
\text { for MBS }\end{array}$ & - \\
\hline$P_{m 0}$ & $\begin{array}{l}\text { Static power expenditures } \\
\text { of MBS }\end{array}$ & $130 \mathrm{~W}$ \\
\hline$P_{f 0}$ & $\begin{array}{l}\text { Static power expenditures } \\
\text { of FAP }\end{array}$ & $4.8 \mathrm{~W}$ \\
\hline$\Delta M$ & $\begin{array}{l}\text { Slope of the load-dependent } \\
\text { power consumption in MBS }\end{array}$ & 4.7 \\
\hline$\Delta F$ & $\begin{array}{l}\text { Slope of the load-dependent } \\
\text { power consumption in FAP }\end{array}$ & 8 \\
\hline$P$ & Transmit power of MBS & $48 \mathrm{~dB}_{\mathrm{m}}$ \\
\hline Q & Transmit power of FAP & $15 \mathrm{~dB}_{\mathrm{m}}$ \\
\hline$\gamma_{m}$ & SINR threshold of macro tier & $4.8 \mathrm{~dB}$ \\
\hline$\gamma_{f}$ & SINR threshold of femto tier & $4 \mathrm{~dB}$ \\
\hline$P_{\text {msleep }}$ & $\begin{array}{l}\text { Power consumption of } \\
\text { sleeping MBS }\end{array}$ & $75 W$ \\
\hline$P_{\text {fsleep }}$ & $\begin{array}{l}\text { Power consumption of } \\
\text { sleeping FAP }\end{array}$ & $5 \mathrm{~W}$ \\
\hline$q_{m}, q_{f}$ & $\begin{array}{l}\text { Probabilities of MBS, FAP } \\
\text { operating in random strategy }\end{array}$ & - \\
\hline$E\left\{s_{m}\right\}, E\left\{s_{f}\right\}$ & $\begin{array}{l}\text { Probabilities of MBS, FAP } \\
\text { operating in proposed } \\
\text { strategy }\end{array}$ & - \\
\hline$\varepsilon_{m r}, \varepsilon_{f r}$ & $\begin{array}{l}\text { Macro, Femto success } \\
\text { probability constraints in } \\
\mathrm{RS}(\mathrm{CAM})\end{array}$ & Scenario dependent \\
\hline$\varepsilon_{m r}^{\prime} \varepsilon_{f r}^{\prime}$ & $\begin{array}{l}\text { Macro, Femto success } \\
\text { probability constraints in } \\
\mathrm{RS}(\mathrm{OAM})\end{array}$ & Scenario dependent \\
\hline$\varepsilon_{m p}, \varepsilon_{f p}$ & $\begin{array}{l}\text { Macro, Femto success } \\
\text { probability constraints in } \\
\text { PS(CAM) }\end{array}$ & Scenario dependent \\
\hline$\varepsilon_{m p^{\prime}}^{\prime} \varepsilon_{f p}^{\prime}$ & $\begin{array}{l}\text { Macro, Femto success } \\
\text { probability constraints in } \\
\text { PS(OAM) }\end{array}$ & Scenario dependent \\
\hline
\end{tabular}

However, we can find that MUE coverage probabilities of strategic strategy provided in [11] are less than random strategy with open access mode. These above results indicate that the effects of access mode for femtocell are greater than the proposed sleeping strategy and power control coefficient $\beta_{m}$ in two-tier femtocell networks. So MUE coverage probabilities of our proposed strategy with open access mode reach the greatest values, and MUE coverage probabilities of random strategy with closed access mode reach the least values.
Nevertheless, when $\sigma^{2}>0.1$, the values of probabilities are very small, and we can not find the trends clearly in Figure 4a. Figure 4b shows us the changes of coverage probability when $\sigma^{2}>0.1$. We observe that the results are consistent with Figure $4 \mathrm{a}$; besides, we find that the strategic sleeping strategy and the proposed sleeping strategy also has improvement over random sleeping strategy with the same coefficient of power control, and coverage probability increases with the coefficient of power control.

Figure 5 plots the relationships among power consumption and activity probability of MBS (FAP) and the coefficient of power control $\left(\beta_{m}\right)$ on the condition that $\sigma^{2}=0$. Here, we do not consider the concrete effect of activity level, so the activity probability of MBS (FAP) is denoted as $q_{m}\left(q_{f}\right)$ no matter what kind of sleeping strategy. We observe that when $\beta_{m}$ and $q_{m}$ are fixed, $q_{f}$ takes little effects on power consumption. Furthermore, when $q_{m}$ and $q_{f}$ are fixed, power consumption increases with $\beta_{m}$ apparently. Besides, when $q_{f}$ and $\beta_{m}$ are fixed, power consumption increases noticeably with $q_{m}$, and the more the value of $\beta_{m}$, the more the increase on power consumption.

In all, the effects of power control on energy consumption and activity probability of MBS are greater than the activity probability of FAP. On the circumstance of our proposed sleeping strategy, according to (17) and (19), the relationships among energy consumption and activity probability and the coefficient of power control are the same as the random sleeping strategy.

In order to facilitate the analysis of the effects of activity probability of BS, here, we assume that MBS in activity 1 with probability represented by the $x$-axis, while activity 0 otherwise. Our proposed sleeping strategy can increase MUE coverage probability, while does not have obvious increase in energy efficiency with the different access modes for femtocell under the condition of $\lambda_{f}=100 \lambda_{m}$, and the reason is that there is nearly no change in FUE coverage probability and total power consumption in the proposed sleeping strategy, even if MUE coverage probability increases, which has little contributions to the system throughput. So in order to see the contrast clearly, here, we define $\lambda_{f}=10 \lambda_{m}=$ $0.01 / m^{2}, \beta_{m}=1$, and the activity probability of each FAP is 0.5 .

Energy efficiency comparisons with different sleeping strategies are showed in Figure 6. We can see that energy efficiency first decreases with $q_{m}$, then tends to flatten, and energy efficiency for the proposed sleeping strategy is higher than other strategies. The reason is that energy consumption of the network increases with the increasing proportion of MBS being active, even if there has less increase in the system throughput, energy efficiency still presents a downward trend, until the proportion approached 1. Moreover, compared with the random 


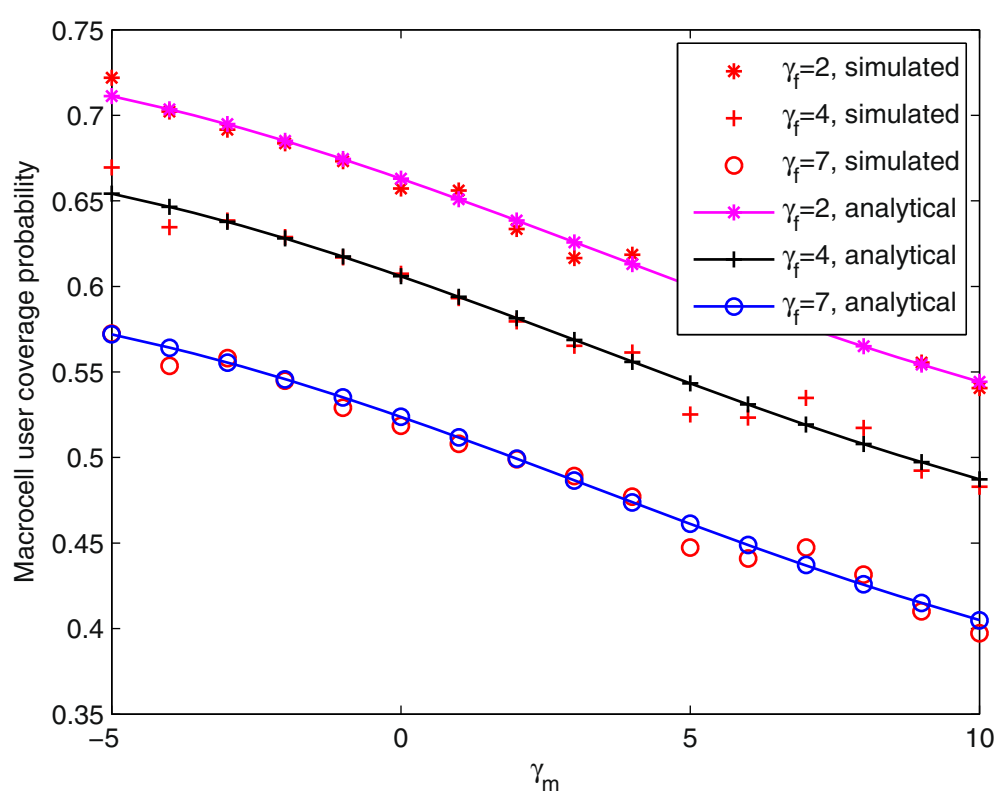

Figure 3 Comparison of analytical results and simulated results for proposed sleeping strategy.

sleeping strategy, there is an increase in the coverage probability for strategic sleeping strategy, so energy efficiency for the strategic sleeping strategy is higher than that for the random sleeping strategy.

\section{Conclusions}

This paper proposes an energy-efficient design of two-tier femtocell network through deploying an activity-aware sleeping strategy in cognitive MBSs and FAPs and analyzes the relationship between them. Based on a stochastic geometry model, the coverage probability of UE, total power consumption, and energy efficiency are derived with different sleeping strategies and access policies of femtocell in the downlink of the two-tier femtocell network. Furthermore, power consumption are formed into optimization problems, which is determined by the a

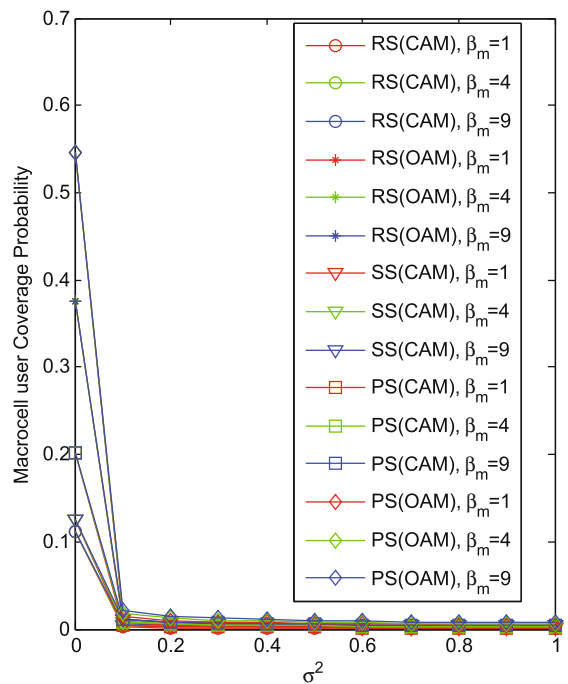

b

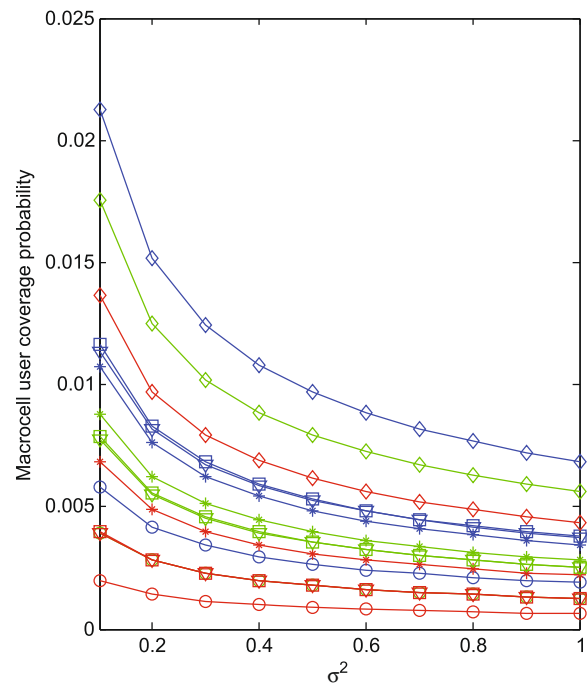

Figure 4 MUE coverage probability for different sleeping strategies and access modes of femtocell. (a) MUE coverage probability for different sleeping strategies and access modes when $0 \leq \sigma^{2} \leq 1$. (b) MUE coverage probability for different sleeping strategies and access modes when $0.1 \leq \sigma^{2} \leq 1$. 


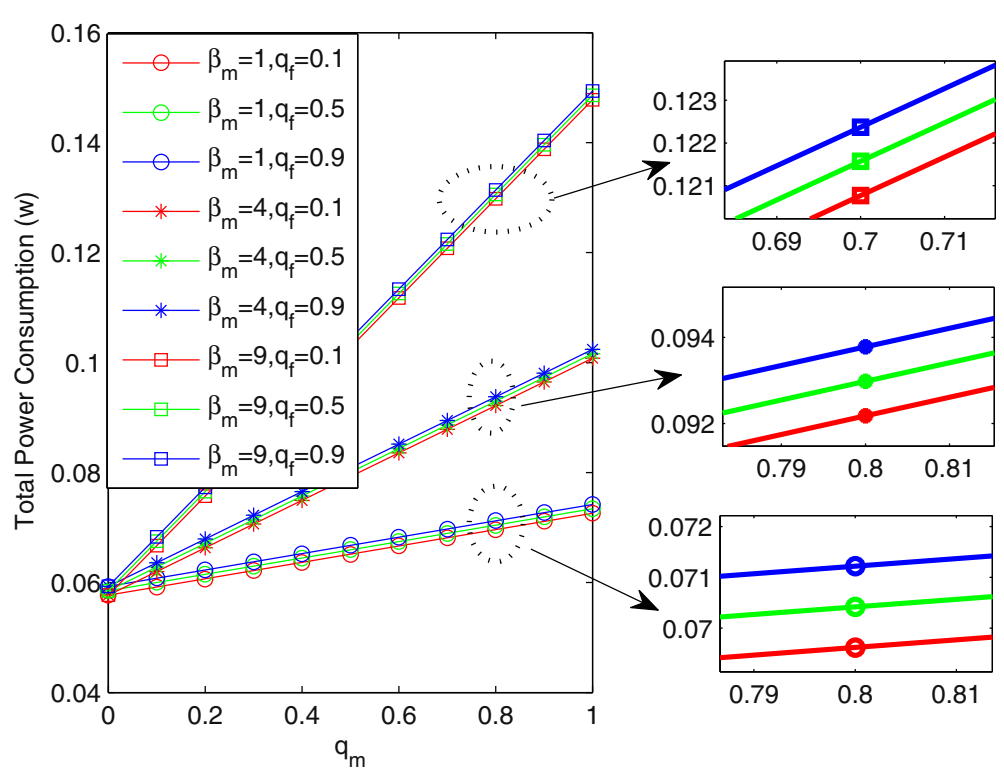

Figure 5 Effects of power control on power consumption.

optimal probabilities of MBS and FAP remain to be active. In addition, the impact of coefficient of power control on power consumption is also investigated. Numerical results show that the proposed sleeping strategy can effectively decrease power consumption and increase energy efficiency.

Completely open or closed access policy may limit the flexibility and performance of network. In the future work, it would be very meaningful to explore energy efficiency scheme in partly open channel of femtocell. Moreover, network power consumption and energy efficiency here only depend on the status of BSs, and user terminal should also be taken into account.

\section{Endnotes}

${ }^{\mathrm{a}}$ Constant $\kappa$ satisfies the equation $0<\kappa<1$ and the value of $\kappa$ affects user's choice. Details refer to [15].

${ }^{\mathrm{b}}$ According to [16] we known that $\mathcal{L}_{I}(r)$ can be simplified to $\exp \left(-\pi r^{2} \lambda C(\alpha)\left(\gamma P_{I} / P_{T}\right)^{2 / \alpha}\right)$ or

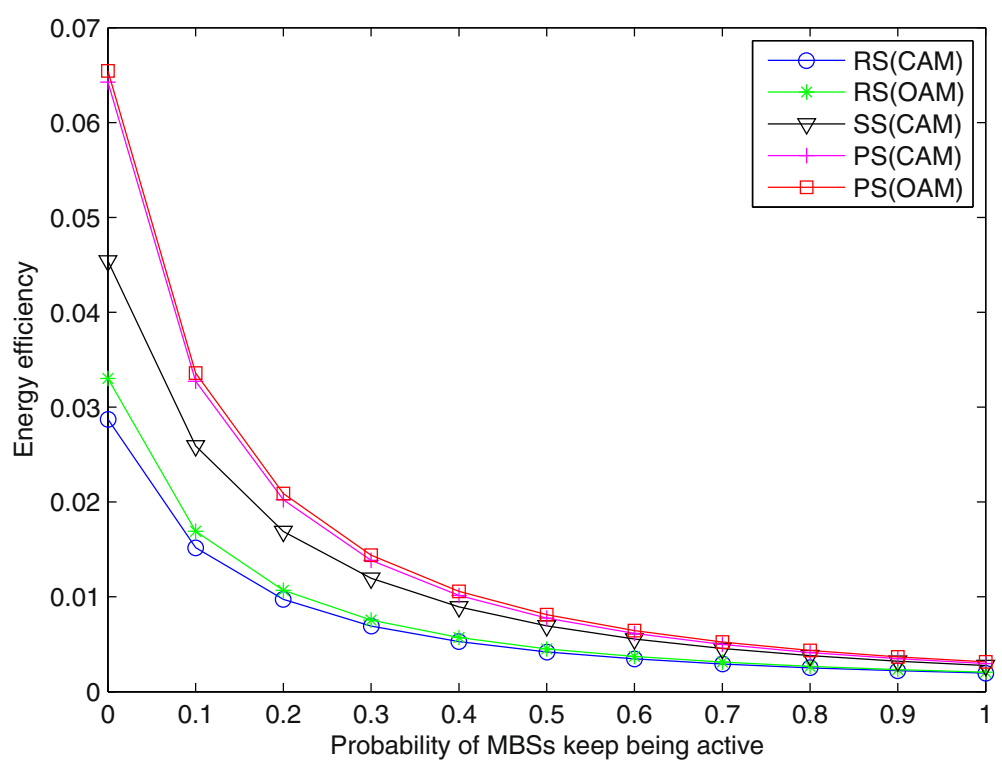

Figure 6 Energy efficiency for different sleeping strategies. 
$\exp \left(-\pi r^{2} \lambda\left(P_{I} / P_{T}\right)^{2 / \alpha} \rho(\gamma, \alpha)\right)$, specific explanations can be found in the reference. For we assume that all MBSs are in sleeping mode, so the interferers all come from FAPs, then $\mathcal{L}_{I}(r)$ is expressed as $\exp \left(-\pi r^{2} \lambda_{f} q_{f}\left(\gamma_{f}\right)^{2 / \alpha} C(\alpha)\right)$.

\section{Appendix 1}

When femtocell operates in open access mode, the coverage probability of MUE includes two parts: MUE connects with MBS and connects with FAP. The access probability of MBS and FAP are $P(M)=\frac{q_{m} \lambda_{m}}{\kappa^{2} \lambda_{f} q_{f}+q_{m} \lambda_{m}}$ and $P(F)=$ $\frac{\kappa^{2} q_{f} \lambda_{f}}{\kappa^{2} \lambda_{f} q_{f}+q_{m} \lambda_{m}}$ on the event that $\kappa r_{m}<r_{f}[18]$.

The coverage probability of a randomly located MUE is taken in (31):

$$
\begin{aligned}
& P_{f o, r s}^{m}=P_{f o, r s}^{m}(M) P(M)+P_{f o, r s}^{m}(F) P(F) \stackrel{a}{=} \int_{r=0}^{\infty} E_{\Phi_{1}, \Phi_{2}} \\
& \times\left[\exp \left(-\frac{r^{\alpha} \gamma_{m}}{P}\left(\sum_{i \in \Phi_{1} \backslash i_{0}} P h_{i} i^{-\alpha}+\sum_{j \Phi_{2}} Q h_{j} j^{-\alpha}\right)\right)\right] \\
& \times f_{R_{m} \mid M}(r) d r \times P(M)+\int_{r=0}^{\infty} E_{\Phi_{1}, \Phi_{2}} \\
& \times\left[\exp \left(-\frac{r^{\alpha} \gamma_{f}}{P_{f}}\left(\sum_{i \in \Phi_{1}} P_{m} h_{i} i^{-\alpha}+\sum_{j \Phi_{2} \backslash j_{0}} Q h_{j} j^{-\alpha}\right)\right)\right] \\
& \times f_{R_{f} \mid F}(r) d r \times P(F)=\int_{r=0}^{\infty} \mathcal{L}_{I}(r) \mathcal{L}_{N}(r) f_{R_{m} \mid M}(r) d r \\
& \times \frac{q_{m} \lambda_{m}}{\kappa^{2} \lambda_{f} q_{f}+q_{m} \lambda_{m}}+\int_{r=0}^{\infty} \mathcal{L}_{I}(r) \mathcal{L}_{N}(r) f_{R_{f} \mid F}(r) d r \\
& \times \frac{\kappa^{2} q_{f} \lambda_{f}}{\kappa^{2} \lambda_{f} q_{f}+q_{m} \lambda_{m}} \stackrel{b}{=} \int_{r=0}^{\infty} \exp \left(-\pi r^{2} q_{m} \lambda_{m} \rho\left(\gamma_{m}, \alpha\right)\right) \\
& \times \exp \left(-\pi r^{2} \kappa^{2} q_{f} \lambda_{f} \rho\left(\frac{\gamma_{m} Q}{\kappa^{\alpha} P}, \alpha\right)\right) \exp \left(-\frac{r^{\alpha} \gamma_{m} \sigma^{2}}{\beta_{m} P}\right) \\
& \times 2 \pi r\left(q_{m} \lambda_{m}+q_{f} \lambda_{f} \kappa^{2}\right) \exp \left(-\pi r^{2}\left(q_{m} \lambda_{m}+q_{f} \lambda_{f} \kappa^{2}\right)\right) d r \\
& \times \frac{q_{m} \lambda_{m}}{\kappa^{2} \lambda_{f} q_{f}+q_{m} \lambda_{m}}+\int_{r=0}^{\infty} \exp \left(-\pi r^{2} q_{f} \lambda_{f} \rho\left(\gamma_{f}, \alpha\right)\right) \\
& \times \exp \left(-\pi\left(\frac{r}{\kappa}\right)^{2} q_{m} \lambda_{m} \rho\left(\frac{\gamma_{f} P \kappa^{\alpha}}{Q}, \alpha\right)\right) \exp \left(-\frac{r^{\alpha} \gamma_{f} \sigma^{2}}{Q}\right) \\
& \times 2 \pi r\left(\frac{q_{m} \lambda_{m}}{\kappa^{2}}+q_{f} \lambda_{f}\right) \exp \left(-\pi r^{2}\left(\frac{q_{m} \lambda_{m}}{\kappa^{2}}+q_{f} \lambda_{f}\right)\right) d r \\
& \times \frac{\kappa^{2} \lambda_{f} q_{f}}{\kappa^{2} \lambda_{f} q_{f}+q_{m} \lambda_{m}} .
\end{aligned}
$$

In (a), $\Phi_{1}$ and $\Phi_{2}$ represent the sets of BSs in macrocell tier and femtocell tier, respectively, and (b) followed by the independence of the poisson distribution, Slivnyak's theorem, and Laplace transform of interference $[5,6]$.

\section{Appendix 2}

In open access mode, the steps of calculating coverage probability are parted into the events of the nearest MBS/FAP being awake and the nearest MBS/FAP being asleep. When $\sigma^{2}=0$, the coverage probability of the active MUE can be expressed as:

$$
P_{f o, p s}^{m}=P_{f o, p s}^{m}(M) P(M)+P_{f o, p s}^{m}(F) P(F) .
$$

$P_{f o, p s}^{m}(M) P(M)$ in (32), denoted as the situation of connecting with MBS, can be written as (33):

$$
\begin{aligned}
& P_{f o, p s}^{m}(M) P(M)=\frac{1}{E\left\{a_{m}\right\}} \int_{0}^{1} x P\left(\operatorname{SINR}>\gamma_{m} \mid x\right) f_{A_{m}}(x) d x \\
& \times \frac{\lambda_{m} E\left\{s_{m}\right\}}{\kappa^{2} \lambda_{f} E\left\{s_{f}\right\}+\lambda_{m} E\left\{s_{m}\right\}} \stackrel{(c)}{\Rightarrow} \frac{1}{E\left\{a_{m}\right\}} \\
& \int_{0}^{1}\left\{x s_{m}(x) P\left(\operatorname{SINR}>\gamma_{m} \mid N_{m}=1\right)\right. \\
& \left.+x\left(1-s_{m}(x)\right) P\left(\operatorname{SINR}>\gamma_{m} \mid N_{m}>1\right)\right\} f_{A}(x) d x \\
& \times \frac{\lambda_{m} E\left\{s_{m}\right\}}{\kappa^{2} \lambda_{f} E\left\{s_{f}\right\}+\lambda_{m} E\left\{s_{m}\right\}}=\frac{1}{E\left\{a_{m}\right\}} \int_{0}^{1} x s_{m}(x) \\
& \int_{r=0}^{\infty} 2 \pi r\left(\lambda_{m}+\lambda_{f} \kappa^{2}\right) \exp \left(-\pi r^{2}\left(\lambda_{m}+\lambda_{f} \kappa^{2}\right)\right) \\
& \times \exp \left(-\pi r^{2} \lambda_{m} E\left\{s_{m}\right\} \rho\left(\gamma_{m}, \alpha\right)\right) \\
& \times \exp \left(-\pi r^{2} \lambda_{f} E\left\{s_{f}\right\} \kappa^{2} \rho\left(\frac{\gamma_{m} Q}{\kappa^{\alpha} P}, \alpha\right)\right) \\
& \times \exp \left(-\frac{r^{\alpha} \gamma_{m} \sigma^{2}}{\beta_{m} P}\right) d r f_{A_{m}}(x) d x \\
& \times \frac{E\left\{s_{m}\right\} \lambda_{m}}{\kappa^{2} \lambda_{f} E\left\{s_{f}\right\}+E\left\{s_{m}\right\} \lambda_{m}}+\frac{1}{E\left\{a_{m}\right\}} \\
& \int_{0}^{1} x\left(1-s_{m}(x)\right) \sum_{i=2}^{\infty} E\left\{s_{m}\right\}\left(1-E\left\{s_{m}\right\}\right)^{i-2} \\
& \int_{r=0}^{\infty} 2\left(\pi\left(\lambda_{m}+\kappa^{2} \lambda_{f}\right)\right)^{i}{ }^{2 i-1} \exp \left(-\pi r^{2}\left(\lambda_{m}+\kappa^{2} \lambda_{f}\right)\right) \\
& \times \exp \left(-\pi r^{2} \lambda_{m} E\left\{s_{m}\right\} \rho\left(\gamma_{m}, \alpha\right)\right) \exp \left(-\frac{r^{\alpha} \gamma_{m} \sigma^{2}}{\beta_{m} P}\right) \\
& \times \exp \left(-\pi r^{2} \lambda_{f} E\left\{s_{f}\right\} \kappa^{2} \rho\left(\frac{\gamma_{m} Q}{\kappa^{\alpha} P}, \alpha\right)\right) \\
& \times d r f_{A_{m}}(x) d x \times \frac{\lambda_{m} E\left\{s_{m}\right\}}{\kappa^{2} \lambda_{f} E\left\{s_{f}\right\}+\lambda_{m} E\left\{s_{m}\right\}} .
\end{aligned}
$$


In (33), (c) follows from reference 5. $P_{f o, p s}^{m}(F) P(F)$ denoted as the situation of connecting with FAP can be taken as in (34):

$$
\begin{aligned}
& P_{f o, p s}^{m}(F) P(F)=\frac{1}{E\left\{a_{m}\right\}} \int_{0}^{1} x P\left(\operatorname{SINR}>\gamma_{f} \mid x\right) f_{A_{m}}(x) d x \\
& \times \frac{\lambda_{f} E\left\{s_{f}\right\} \kappa^{2}}{\lambda_{f} E\left\{s_{f}\right\} \kappa^{2}+\lambda_{m} E\left\{s_{m}\right\}}=\frac{1}{E\left\{a_{m}\right\}} \\
& \int_{0}^{1}\left\{x s_{f}(x) P\left(\operatorname{SINR}>\gamma_{f} \mid N_{f}=1\right)\right. \\
& \left.+x\left(1-s_{f}(x)\right) P\left(\operatorname{SINR}>\gamma_{f} \mid N_{f}>1\right)\right\} f_{A_{m}}(x) d x \\
& \times \frac{\lambda_{f} E\left\{s_{f}\right\} \kappa^{2}}{\lambda_{f} E\left\{s_{f}\right\} \kappa^{2}+\lambda_{m} E\left\{s_{m}\right\}}=\frac{1}{E\left\{a_{m}\right\}} \\
& \int_{0}^{1} x s_{f}(x) \int_{r=0}^{\infty} 2 \pi r\left(\lambda_{f}+\frac{\lambda_{m}}{\kappa^{2}}\right) \\
& \times \exp \left(-\pi r^{2}\left(\lambda_{f}+\frac{\lambda_{m}}{\kappa^{2}}\right)\right) \exp \left(-\pi r^{2} \lambda_{f}\right. \\
& \left.\times E\left\{s_{f}\right\} \rho\left(\gamma_{f}, \alpha\right)\right) \exp \left(-\pi\left(\frac{r}{\kappa}\right)^{2} \lambda_{m} E\left\{s_{m}\right\}\right. \\
& \left.\times \rho\left(\frac{\gamma_{f} P \kappa^{\alpha}}{Q}, \alpha\right)\right) \exp \left(-\frac{r^{\alpha} \gamma_{f} \sigma^{2}}{Q}\right) d r f_{A_{m}}(x) d x \\
& \times \frac{\lambda_{f} E\left\{s_{f}\right\} \kappa^{2}}{\lambda_{f} E\left\{s_{f}\right\} \kappa^{2}+\lambda_{m} E\left\{s_{m}\right\}}+\frac{1}{E\left\{a_{m}\right\}} \\
& \int_{r=0}^{\infty} x\left(1-s_{f}(x)\right) \sum_{i=2}^{\infty} E\left\{s_{f}\right\}\left(1-E\left\{s_{f}\right\}\right)^{i-2} \\
& \int_{r=0}^{\infty} 2\left(\pi\left(\lambda_{f}+\frac{\lambda_{m}}{\kappa^{2}}\right)\right)^{i} r^{2 i-1} \\
& \times \exp \left(-\pi r^{2}\left(\lambda_{f}+\frac{\lambda_{m}}{\kappa^{2}}\right)\right) \exp \left(-\pi r^{2} \lambda_{f}\right. \\
& \left.\times E\left\{s_{f}\right\} \rho\left(\gamma_{f}, \alpha\right)\right) \exp \left(-\pi\left(\frac{r}{\kappa}\right)^{2} \lambda_{m} E\left\{s_{m}\right\}\right. \\
& \left.\times \rho\left(\frac{\gamma_{f} P \kappa^{\alpha}}{Q}, \alpha\right)\right) \exp \left(-\frac{r^{\alpha} \gamma_{f} \sigma^{2}}{Q}\right) \\
& \times d r f_{A_{m}}(x) d x \times \frac{\lambda_{f} E\left\{s_{f}\right\} \kappa^{2}}{\lambda_{f} E\left\{s_{f}\right\} \kappa^{2}+\lambda_{m} E\left\{s_{m}\right\}} .
\end{aligned}
$$

Substituting (33) and (34) into (32), when $\sigma^{2}=0$, the expression can be simplified to (16).

\section{Competing interests}

The authors declare that they have no competing interests.

\section{Acknowledgements}

This work is supported by National Key Project (2013ZX03001025-002) National 863 Project (2014AA01A701), and National Nature Science Foundation of China $(61372113,61431003,61421061)$

Received: 30 June 2014 Accepted: 5 January 2015

Published online: 24 February 2015

\section{References}

1. V Jungnickel, K Manolakis, W Zirwas, B Panzner, V Braun, M Lossow, M Sternad, R Apelfrojd, T Svensson, The role of small cells, coordinated multipoint, and massive MIMO in 5G. IEEE Commun. Mag. 52(5), 44-51 (2014)

2. $X \mathrm{Ge}, \mathrm{TH}$ Han, Y Zhang, Spectrum and energy efficiency evaluation of two-tier Femtocell networks with partially open channels. IEEE Trans. Vehicular Technol. 63(3), 1306-1319 (2014)

3. Z Hasan, H Boostanimehr, VK Bhargava, Green cellular networks: a survey, some research issues and challenges. IEEE Commun. Surveys Tutorials. 13(4), 524-540 (2011)

4. G Auer, V Giannini, C Desset, I Godor, P Skillermark, M Olsson, MA Imran, D Sabella, MJ Gonzalez, O Blume, A Fehske, How much energy is needed to run a wireless network? IEEE Wireless Commun. Mag. 18(5), 40-49 (2011)

5. WD Stoyan, J Mecke, Stochastic geometry and its applications, 2nd ed. (John Wiley and Sons, New York, 2008)

6. M Haenggi, Stochastic geometry for wireless networks. (Cambridge University, England, 2012)

7. I Ashraf, F Boccardi, L Ho, Sleep mode techniques for small cell deployments. IEEE Commun. Mag. 49(8), 72-79 (2011)

8. M Wildemeersch, TQS Quek, A Rabbachin, CH Slump, A Huang, in IEEE International Conference on Communications (ICC). Energy effcient design of cognitive small cells (IEEE Budapest, Hungary, 2013), pp. 1294-1299

9. M Wildemeersch, TQS Quek, A Rabbachin, CH Slump, A Huang, in IEEE Wireless Communications and Networking Conference (WCNC). Performance limits for cognitive small cells (IEEE Shanghai, China, 2013), pp. 2832-2836

10. J Peng, P Hong, KXue, Stochastic analysis of optimal base station energy saving in cellular networks with sleep mode. IEEE Commun. Lett. 18(4), 612-615 (2014)

11. YS Soh, TQS Quek, M Kountouris, H Shin, Energy effcient heterogeneous cellular networks. IEEE J. Sel. Areas Commun. 31(5), 840-850 (2013)

12. Z Pan, S Shimamoto, in IEEE Wireless Communications and Networking Conference (WCNC). Cell sizing based energy optimization in joint Macro-Femto deployments via sleep activation (IEEE Shanghai, China, 2013), pp. 4765-4770

13. D Cao, S Zhou, Z Niu, Optimal combination of base station densities for energy-efficient two-tier heterogeneous cellular networks. IEEE Trans. Wireless Commun. 12(9), 4350-4362 (2013)

14. YS Soh, TQS Quek, M Kountouris, G Caire, Cognitive hybrid division duplex for two-tier femtocell networks. IEEE Trans. Wireless Commun. 12(10), 4852-4865 (2013)

15. H ElSawy, E Hossain, HetNets with cognitive small cells: user offloading and distributed channel access techniques. IEEE Commun. Mag. 51(6), 28-36 (2013)

16. F Baccelli, B Blaszczyszyn, P Muhlethaler, Stochastic analysis of spatial and opportunistic ALOHA. IEEE J. Sel. Areas Commun. 27(7), 1105-1119 (2009)

17. WC Cheung, TQS Quek, M Kountouris, Throughput optimization, spectrum sharing, and femtocell access in two-tier femtocell networks. IEEE J. Selected Areas Commun. 30(3), 561-574 (2012)

18. V Chandrasekhar, JG Andrews, Spectrum allocation in tiered cellular networks. IEEE Trans. Commun. 57(10), 3059-3068 (2009)

\section{Submit your manuscript to a SpringerOpen ${ }^{\circ}$ journal and benefit from:}

- Convenient online submission

- Rigorous peer review

- Immediate publication on acceptance

- Open access: articles freely available online

- High visibility within the field

- Retaining the copyright to your article

Submit your next manuscript at $>$ springeropen.com 